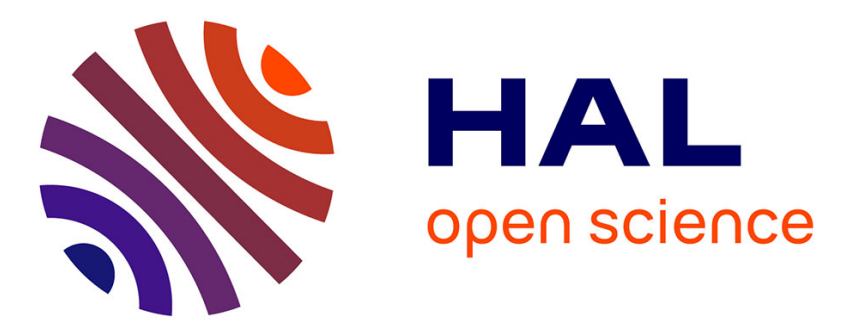

\title{
A positive to negative uniaxial thermal expansion crossover in an organic benzothienobenzothiophene structure
}

Dan Dumitrescu, Gilles Roche, Joël Moreau, Olivier Dautel, Arie van Der Lee

\section{- To cite this version:}

Dan Dumitrescu, Gilles Roche, Joël Moreau, Olivier Dautel, Arie van Der Lee. A positive to negative uniaxial thermal expansion crossover in an organic benzothienobenzothiophene structure. Acta Crystallographica Section B : Structural Science, Crystal Engineering and Materials [2014-...], 2020, 76 (4), pp.661-673. 10.1107/S2052520620007702 . hal-02935116

\section{HAL Id: hal-02935116 \\ https://hal.umontpellier.fr/hal-02935116}

Submitted on 10 Nov 2020

HAL is a multi-disciplinary open access archive for the deposit and dissemination of scientific research documents, whether they are published or not. The documents may come from teaching and research institutions in France or abroad, or from public or private research centers.
L'archive ouverte pluridisciplinaire HAL, est destinée au dépôt et à la diffusion de documents scientifiques de niveau recherche, publiés ou non, émanant des établissements d'enseignement et de recherche français ou étrangers, des laboratoires publics ou privés. 


\section{A positive to negative uniaxial thermal expansion crossover in an organic benzothienobenzothiophene structure}

Authors

Dan G. Dumitrescua ${ }^{\mathrm{a}}$, Gilles H. Roche ${ }^{\mathrm{bc}}$, Joël J. E. Moreau ${ }^{\mathrm{b}}$, Olivier J. Dautel ${ }^{\mathrm{b}}$ and Arie van der Lee $^{\mathrm{d} *}$

aElettra, Sincrotrone Trieste S.C.p.A., Strada Statale 14 - km 163,5 in AREA Science Park, Basovizza, Trieste, 34149, Italy

' ICGM, Université de Montpellier, CNRS, ENSCM, Montpellier, France

'Université de Bordeaux, IMS, CNRS, UMR 5218, Bordeaux INP, ENSCBP, 33405, Talence, 33405, France

Institut Européen des Membranes, IEM - UMR 5635, ENSCM, CNRS, Université de Montpellier, Montpellier, France

Correspondence email: arie.van-der-lee@umontpellier.fr

Synopsis A rare transition from positive to negative thermal expansion in an organic herringbone stacked structure is reported and related to an isosymmetric phase transition.

Abstract 4,4'-(benzo[b]benzo[4,5]thieno[2,3- $d]$ thiophene-2,7-diyl)bis(butan-1-ol), BTBT-C4OH, displays a continuous type 0 first-order isosymmetric phase transition at $200 \mathrm{~K}$ which is accompanied by a continuous change of the thermal expansion along the $b$-axis from positive to negative. The equivalent isotropic atomic displacement parameters for all non-hydrogen atoms as well as all the eigenvalues of the anisotropic atomic displacement tensor show discontinuous behavior at the phase transition. The eigenvalues of the translational tensor in a rigid-body description of the molecule are all discontinuous at the phase transition, but the librational eigenvalues are discontinuous only in their temperature derivative. BTBT-C4OH displays a similar type of quasi-supercritical phase transition as BTBT-C6OH, despite the difference in molecular packing and the very large difference in thermal expansion magnitudes.

Keywords: Isosymmetric phase transition, thermal expansion, organic structure

\section{Introduction}

The [1]benzothieno[3,2-b][1]-benzothiophene (BTBT) $\pi$-conjugated system is one the best performing organic semiconductors for use in thin film transistors with record hole $\left(\mathrm{h}^{+}\right)$mobilities 
Yuan et al., 2014; Cho et al., 2015). The symmetric or asymmetric functionalizing of the BTBT system serves to increase the charge carrier mobility and to covalently link receptors for chemical sensing. BTBT derivatives with alkyl chains at the 2 and 7 positions have been reported (Izawa et al., 2008) but also more with bulky fragments (Iino et al., 2015; Schweicher et al., 2015). Roche et al. (2016) described the symmetric funtionalization of the BTBT core by a hydroxy-hexyl chain, thereby offering relatively strong tethering attachments between individual molecules. Thermally evaporated thin film field effect transitors (OFET) based on this diol derivative gave excellent mobilities of $0.17 \mathrm{~cm}^{2} \mathrm{~V}^{-1} \mathrm{~s}^{-1}$ at room temperature.

BTBT-C4OH, 4,4'-(benzo[ $b$ ]benzo[4,5]thieno[2,3- $d$ ] thiophene-2,7-diyl)bis(butan-1-ol), is a short chain member of the BTBT-CnOH family of symmetrically functionalized $\pi$-conjugated BTBT systems, of which especially long chain members present excellent semiconducting properties for OFET applications (Dautel et al., 2020). The X-ray structure of the $n=6$ member, BTBT-C6OH, was published before (Roche et al., 2016) and was shown to display, in addition to its good mobility characteristics, colossal negative uniaxial thermal expansion along the monoclinic $b$-axis, one of the largest negative expansivities in organic compounds observed until now (van der Lee et al., 2018). The compound was also shown to have an isosymmetric phase transition near $210 \mathrm{~K}$. The negative thermal expansion in BTBT-C6OH is related to a compensation mechanism caused by steric hindrance between adjacent molecules and enhanced by moderate anharmonity in the low-temperature mother phase and strong anharmonicity in the mixed high-temperature phase. Isosymmetric phase transitions are rare, but not unprecedented (Katrusiak, 2001). Examples include the 3,5pyridinedicarboxylic acid structural system (Ford et al., 2011), for which the experimental diffraction data only could be modeled supposing a nucleation of a high-temperature (HT) inside a lowtemperature (LT) matrix until complete co-existence near the phase transition temperature, after which the LT phase slowly disappears at the expense of the HT phase. Another well-described case is the inorganic scheelite $\mathrm{Li}_{0.5} \mathrm{Ce}_{0.5} \mathrm{MoO}_{4}$, for which diffraction and Raman spectroscopy (Saha et al., 2013) showed a softening of the zone center phonons towards the first-order phase transition, and also a high-temperature phase growing in the low-temperature phase, but without a complete co-existence near the phase transition or complete disappearance beyond the phase transition temperature. In the case of BTBT-C6OH the relative proportions of the LT and HT phases could not be determined from the experimental diffraction data, but were followed by ab initio molecular dynamics showing equal proportions of the two phases in the HT region.

Isosymmetric phase transitions are necessarily first-order and should have a discontinuity in the first derivative of the free energy with respect to a thermodynamic variable, which is in practice very often expressed as a discontinuity in cell volume and one or more cell parameters as a function of temperature or pressure. In the case of BTBT-C6OH no discontinuity in the cell parameters could be observed, but discontinuities in derived secondary geometrical parameters such as the slope of the 
temperature dependence of the angle between adjacent benzothieno-benzothiophene (BTBT) moieties were established. In analogy with the supercritical transition from a liquid to a gas where there is no discontinuity of the density, combined with the results from the $a b$ initio molecular dynamics study, the transition in BTBT-C6OH was classified as a pseudo supercritical first-order phase transition. These cases have been in particular addressed by Christy (1995), who labeled this type of transitions as continuous first-order type 0 structural phase transitions. The properties of the Widom line in crystalline solids with continuous supercritical transitions were recently addressed by Tareyeva et al. (2018).

In the search of other similar compounds having comparable exceptional thermal expansion properties or good OFET characteristics such as in BTBT-C6OH, the compound BTBT-C4OH was synthesized and its structure determined by X-ray diffraction. In this paper we report the evolution of the structural parameters of BTBT-C4OH as a function of temperature. Despite a different relative orientation of the BTBT moieties with respect to that in BTBT-C6OH a similar continuous type 0 phase transition takes place near $200 \mathrm{~K}$. The compound does not have colossal positive or negative thermal expansion properties, but displays a continuous crossover from positive to negative uniaxial expansion at the phase transition temperature. This switching from positive to negative thermal expansion in an one-component organic compound without discontinuous phase transition is nearly unprecedented: only one report was found for the closely related compounds 1,4-bis(S-3-butyn-2-ol)benzene and 2,6-bis(S-3-butyn2-ol)-naphthalene (Negi et al., 2018), describing quasi-continuous thermally induced crossovers near 270 and $300 \mathrm{~K}$, respectively. A rare case of a continuous isosymmetric phase transition accompanied by a positive to negative thermal expansion crossover in a hybrid AlNa bimetallic ammonium metal formate framework structure was reported by $\mathrm{Yu}$ et al. (2017).

\section{Synthesis and crystal growth}

\subsection{General}

All chemicals and solvents are of reagent grade. Dichloromethane (DCM), N,N-dimethylformamide, (DMF), was dried over $4 \AA$ molecular sieves, acetic acid over $3 \AA$ molecular sieves and tetrahydrofuran (THF) was distilled under nitrogen over sodium benzophenone ketyl. $1 \mathrm{H}$ and $13 \mathrm{C}$ NMR spectra were recorded on a Bruker AC 400MHz spectrometer. Chemical shifts (d), reported in parts per million and are relative to tetramethylsilane. Signal multiplicities are noted by s (singlet), d (doublet), dd (doublet of doublet), $\mathrm{t}$ (triplet), $\mathrm{tt}$ (triplet of triplet), $\mathrm{m}$ (multiplet). MS spectra were obtained on a Synapt G2-S from Waters with a quadrupole cell collision. The NMR and mass spectra of the intermediate and final products are reported in the Supplementary Material (Figs S5-S13). 


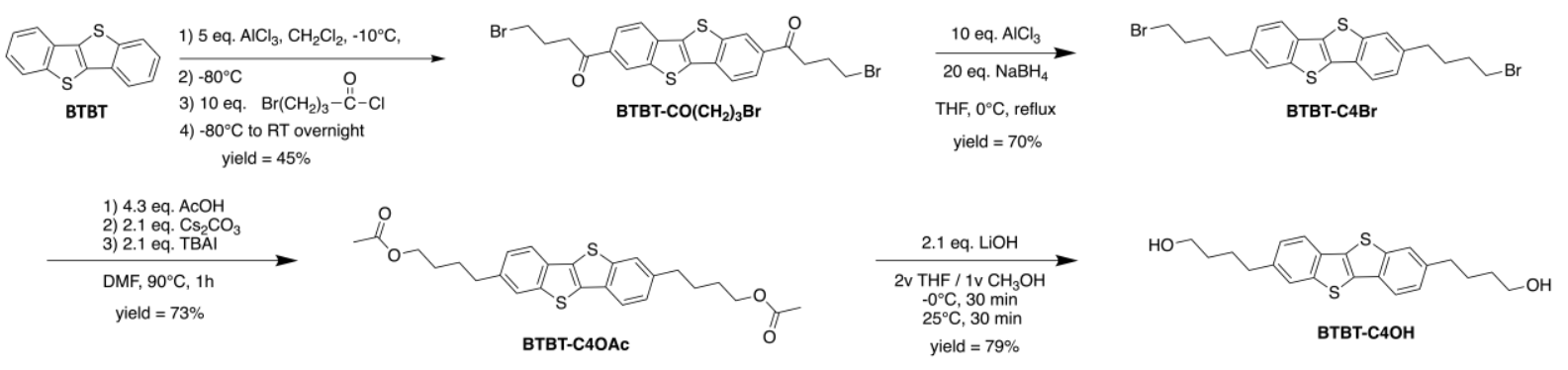

Scheme 1: Synthetic pathways to BTBT-C4OH

\subsection{Synthetic procedures}

The synthesis of the diol BTBT-C4OH was adapted from the synthetic pathway that we described for BTBT-C6OH (Roche et al., 2016). In a first step, the BTBT core was functionalized by a Friedel and Craft acylation at the positions 2 and 7 to afford the BTBT-CO(C $\left(\mathbf{C H}_{2}\right)_{3} \mathbf{B r}$. The side products of this step should be acylated BTBT derivatives at the position 2,8; 1,7 and 2,9. All of them were easily removed by two recrystallizations in chlorobenzene. The keto functions were reduced by a mixture of $\mathrm{AlCl}_{3}$ and $\mathrm{NaBH}_{4}$ before the introduction of the oxygen atoms by a SN2 reaction with in situ prepared cesium acetate assisted by tetrabutylammonium iodide. Finally, the diol BTBT-C4OH was obtained by a saponification of the acetyl functions by two equivalents of lithium hydroxide.

\subsubsection{1,1'-(benzo[b]benzo[4,5]thieno[2,3- $d$ ] thiophene-2,7-diyl)bis(4-bromobutan-1-one) (BTBT- $\left.\mathrm{CO}\left(\mathrm{CH}_{2}\right)_{3} \mathrm{Br}\right)$ :}

$1.2 \mathrm{~g}$ (1 eq., $5 \mathrm{mmol}$ ) of BTBT were dissolved in $120 \mathrm{~mL}$ of dry DCM under nitrogen. The solution was cooled to $-10{ }^{\circ} \mathrm{C}$ (a small amount of BTBT reprecipitated). $3.7 \mathrm{~g}$ of aluminium chloride (5 eq., 25 mmol) were then added and the mixture took a red-brown color. After cooling to $-84{ }^{\circ} \mathrm{C}, 9.7 \mathrm{~g}$ of 6 bromobutanoyl chloride (10 eq., $50 \mathrm{mmol}$ ) were added dropwise. The mixture took yellow-red highlights. The mixture was maintained below $-60^{\circ} \mathrm{C}$ during $2 \mathrm{~h}$ to control the reaction to the monoacylation and slowly raised to room temperature overnight. The mixture was diluted with $1000 \mathrm{~mL}$ of $\mathrm{CH}_{2} \mathrm{Cl}_{2}$ and $500 \mathrm{~mL}$ of water were slowly added, after which the red color turned to yellow. After decantation, the organic phase was heated at $40^{\circ} \mathrm{C}$ in the presence of $\mathrm{MgSO} 4$, and filtrated at this temperature. Finally, the solvent and the excess of the acid chloride were removed to furnish $4.5 \mathrm{~g}$ of crude product. This crude solid was recrystallized twice in $40 \mathrm{~mL}$ of chlorobenzene to furnish $1 \mathrm{~g}$ (yield $=45 \%$ ) of golden glitters after a hot filtration. 1H NMR $\left(\mathrm{CDCl}_{3}\right) \delta: 8.60(\mathrm{dd}, \mathrm{J}=1.5 \mathrm{~Hz}, 0.5$ $\mathrm{Hz}, 2 \mathrm{H}), 8.11(\mathrm{dd}, \mathrm{J}=8.4 \mathrm{~Hz}, 1.5 \mathrm{~Hz}, 2 \mathrm{H}), 8.00(\mathrm{dd}, \mathrm{J}=8.4 \mathrm{~Hz}, 0.5 \mathrm{~Hz}, 2 \mathrm{H}), 3.61(\mathrm{t}, \mathrm{J}=6.2 \mathrm{~Hz}, 4 \mathrm{H})$, $3.32(\mathrm{t}, \mathrm{J}=6.9 \mathrm{~Hz}, 4 \mathrm{H}), 2.39(\mathrm{q}, \mathrm{J}=6,7 \mathrm{~Hz}, 4 \mathrm{H})$; MS HRMS (ASAP+) m/z (MH+) = obsd 536.9189, calc 536.9193.

\subsubsection{2,7-bis(4-bromobutyl)benzo[b]benzo[4,5]thieno[2,3-d]thiophene (BTBT-C4Br):}


$0.9 \mathrm{~g}$ ( 1 eq., $1.67 \mathrm{mmol}$ ) of BTBT-CO(C $\left.\mathbf{C H}_{2}\right)_{3} \mathbf{B r}$ were dissolved in $90 \mathrm{~mL}$ of dry THF under nitrogen and cooled to $0^{\circ} \mathrm{C}$. $2.23 \mathrm{~g}$ (10 eq., $16.7 \mathrm{mmol}$ ) of aluminium chloride and $1.26 \mathrm{~g}$ (20 eq., $33.44 \mathrm{mmol}$ ) of sodium borohydride were successively added portion wise. The temperature was raised to $40^{\circ} \mathrm{C}$ to ignite the reaction. After 30 minutes, the yellow mixture was refluxed for 12 hours and turned colorless. After cooling down to room temperature, the mixture was quenched by $100 \mathrm{~mL}$ of water and the THF was eliminated by distillation under reduced pressure. $200 \mathrm{~mL}$ of DCM were added and the mixture was washed twice with a $\mathrm{NaHCO}_{3} 10 \%$ solution and once by a saturated $\mathrm{NaCl}$ solution. The organic layer was dried over $\mathrm{MgSO}_{4}$ and concentrated under reduced pressure. The crude product was purified by a column chromatography on silica gel using a mixture of cyclohexane and dichloromethane as eluent. $0.36 \mathrm{~g}$ (yield: $70 \%$ ) of white crystals of (BTBT-C4Br) were obtained. ${ }^{1} \mathrm{H}$ $\operatorname{NMR}\left(\mathrm{CDCl}_{3}\right) \delta: 7.78(\mathrm{dd}, \mathrm{J}=8.1 \mathrm{~Hz}, 0.5 \mathrm{~Hz}, 2 \mathrm{H}), 7.71(\mathrm{dd}, \mathrm{J}=1.5 \mathrm{~Hz}, 0.5 \mathrm{~Hz}, 2 \mathrm{H}), 7.27(\mathrm{dd}, \mathrm{J}=8.1$ $\mathrm{Hz}, 1.5,2 \mathrm{H}), 3.45(\mathrm{t}, \mathrm{J}=6.4 \mathrm{~Hz}, 4 \mathrm{H}), 2.80(\mathrm{t}, \mathrm{J}=7.5 \mathrm{~Hz}, 4 \mathrm{H}), 1.94(\mathrm{~m}, 4 \mathrm{H}), 1.87(\mathrm{~m}, 4 \mathrm{H}) ;{ }^{13} \mathrm{C} \mathrm{NMR}$ $\left(\mathrm{CDCl}_{3}\right) \delta: 142.50,138.92,132.67,131.38,125.73,123.36,121.26,35.11,33.56,32.17,30.00 ; \mathrm{MS}$ HRMS $\left(\mathrm{ES}^{+}\right) \mathrm{m} / \mathrm{z}\left(\mathrm{MH}^{+}\right)=$obsd 508.9593, calc 508.9608.

\subsection{3. benzo[ $b]$ benzo[4,5]thieno[2,3- $d]$ thiophene-2,7-diylbis(butane-4,1-diyl) diacetate (BTBT- C4OAc):}

$402.38 \mathrm{mg}$ of cesium carbonate (2.1 eq., $1.25 \mathrm{mmol}$ ) were suspended in $90 \mathrm{ml}$ of dry DMF under nitrogen. $140 \mu \mathrm{L}$ of dry acetic acid (4.3 eq., $2.50 \mathrm{mmol})$ were introduced. A smooth heating was necessary to promote the formation of cesium carboxylate. $300 \mathrm{mg}$ of BTBT-C4Br (1 eq., 0.60) were added followed by $456.2 \mathrm{mg}$ (2.1 eq., 2.1) of tetrabutylammonium iodide. The mixture was stirred at $90{ }^{\circ} \mathrm{C}$ for $1 \mathrm{~h}$. After cooling down to room temperature, the mixture was diluted with $40 \mathrm{~mL}$ of water and the precipitate was isolated by centrifugation. Recrystallisation of the crude solid in $40 \mathrm{~mL}$ of ethanol furnished $200 \mathrm{mg}$ (yield: $73 \%$ ) of white needles of BTBT-C4OAc. ${ }^{1} \mathrm{H}$ NMR $\left(\mathrm{CDCl}_{3}\right) \delta: 7.76$ $(\mathrm{dd}, \mathrm{J}=8.0 \mathrm{~Hz}, 0.4 \mathrm{~Hz}, 2 \mathrm{H}), 7.72(\mathrm{dd}, \mathrm{J}=1.5 \mathrm{~Hz}, 0.4 \mathrm{~Hz}, 2 \mathrm{H}), 7.28(\mathrm{dd}, \mathrm{J}=8.0 \mathrm{~Hz}, 1.5,2 \mathrm{H}), 4.12$ (t, $\mathrm{J}=6.4 \mathrm{~Hz}, 4 \mathrm{H}), 2.80(\mathrm{t}, \mathrm{J}=7.2 \mathrm{~Hz}, 4 \mathrm{H}), 2.06(\mathrm{~s}, 6 \mathrm{H}), 1.75(\mathrm{~m}, 8 \mathrm{H}) ;{ }^{13} \mathrm{C} \mathrm{NMR}\left(\mathrm{CDCl}_{3}\right) \delta: 171.18$, $142.47,139.15,132.67,131.34,125.76,123.36,121.22,64.27,35.57,28.17,27.91,20.99 ;$ MS HRMS $\left(\mathrm{ES}^{+}\right) \mathrm{m} / \mathrm{z}\left(\mathrm{MH}^{+}\right)=$obsd 469.1500, calc 469.1507.

\subsubsection{4,4'-(benzo[b]benzo[4,5]thieno[2,3- $d]$ thiophene-2,7-diyl)bis(butan-1-ol) (BTBT-C4OH):}

$150 \mathrm{mg}$ (1 eq., $0.32 \mathrm{mmol}$ ) of BTBT-C4OAc were suspended in $8 \mathrm{~mL}$ of a mixture of THF and $\mathrm{CH} 3 \mathrm{OH}$ in a 2:1 ratio at room temperature. Increasing the temperature solubilized the BTBT derivative which remained in solution at room temperature (RT). After 10 minutes of nitrogen bubbling, the mixture was cooled to $0^{\circ} \mathrm{C}$ causing the precipitation of the starting material. $16 \mathrm{mg}$ ( 2.1 eq., $0.67 \mathrm{mmol}$ ) of $\mathrm{LiOH}$ were finally added and the temperature maintained at $0^{\circ} \mathrm{C}$ for 30 minutes while the precipitate remained in suspension. Increasing the temperature to room temperature caused the solubilization of the starting material. After 30 minutes at RT, a TLC monitoring, confirmed the 
completion of the reaction which was quenched by the addition of $1 \mathrm{~N} \mathrm{HCl}$ after $2 \mathrm{~h}$ at $\mathrm{RT}$. The solution was diluted with $20 \mathrm{~mL}$ of water, THF and $\mathrm{CH}_{3} \mathrm{OH}$ were removed under vacuum and water was added. The white precipitate was recovered by centrifugation and recrystallized in toluene to furnish $97 \mathrm{mg}$ (yield: 88\%) of translucent crystals of BTBT-C4OH, typically in the form of small sticks. ${ }^{1} \mathrm{H}$ NMR (THF-d8) 8: 7.79 (s, 2H), 7.78 (d, J = 8.4 Hz, 2H), 7.30 (d, J = 8.4 Hz, 2H), 3.51 (dt, $\mathrm{J}=11.6 \mathrm{~Hz}, 5.6 \mathrm{~Hz}, 4 \mathrm{H}), 3.39$ (t, J = 5.6 Hz, 2H), 2.78 (t, J = 7.6 Hz, 4H), 1.78 (m, 4H), 1.57 (m, 4H);

${ }^{13}$ C NMR (THF-d8) $\delta: 140.55,138.26,130.51,129.31,123.97,121.46,118.94,59.52,33.85,30.77$, $30.77,26.17$.

\section{Data collection and data treatment}

The data were collected using a Pilatus 2M detector at the XRD1 beamline of the Elettra synchrotron (Lausi et al., 2015) at a wavelength of $0.7000 \AA .180 \phi$ frames at $1^{\circ}$ interval were taken for each data collection on a Huber $\kappa$-goniometer from 100 to $300 \mathrm{~K}$ in steps of $10 \mathrm{~K}$ and then from $300 \mathrm{~K}$ to $100 \mathrm{~K}$ and again up to $140 \mathrm{~K}$. In total 46 data collections were carried out. The XDS program (Kabsch, 2010ab) was used to integrate the frames and to apply corrections and for each dataset the space group and cell parameters were checked and confirmed using the POINTLESS program from the CCP4 software suite (Winn et al., 2011).

The structure crystallizes in the monoclinic space group $P 2_{1} / c$ and was easily solved using the charging flipping algorithm implemented in SUPERFLIP (Palatinus \& Chapuis, 2007). The structural models were refined using SHELXL (Sheldrick, 2015) with hydrogen atoms riding on their parent atoms, except the hydrogen of the terminal hydroxyl group which position was refined keeping only the C-O-H angle tetrahedrally. All data sets were refined with the same restraints and constraints and appeared to have approximately the same quality, as expressed by $R_{\text {int }}$ which fluctuates between 0.0214 and 0.0292 without any obvious trend with temperature. $R_{\mathrm{F}}(I>2 \sigma(I))$ increased slightly from 0.038 at $100 \mathrm{~K}$ to 0.043 at $300 \mathrm{~K}$. The ratio of reflections with $I>2 \sigma(I)$ to the total number of unique reflections was for all data sets larger than 0.85 . The atomic displacement parameters of all atoms are fairly small and at first sight regular at all temperatures with the largest $U_{\text {eq }}$ value that of $\mathrm{O} 13$ at $300 \mathrm{~K}$ $\left(0.0669(4) \AA^{2}\right.$ ). A closer inspection did, however, show some irregularities in the temperature evolution of the ellipsoids (see below). Not any sign of disorder could be found, nor of overlooked or too much imposed symmetry. Table S1 gives the experimental and refinement details of the first 21 data collection spanning the complete temperature range between 100 and $300 \mathrm{~K}$.

An additional data collection was carried out at the CRISTAL beamline $(\lambda=0.6722 \AA)$ of the Soleil synchrotron (Saint-Aubin, France) at $116 \mathrm{~K}$ at a slightly better resolution $(0.66 \AA)$ than the data from 
Elettra. A 4-circle Newport diffractometer was used for the experiment and the data were recorded on a Rigaku Atlas CCD detector (10.29 pixels/mm resolution). It should be noted that a different crystal than the one used at Elettra was mounted for this experiment. The reflection intensities from the data collection were used for a Hirshfeld atomic refinement (Capelli et al., 2014) with free positional parameters for all atoms, free anisotropic displacement parameters for the non-hydrogen atoms and free isotropic displacement parameters for the hydrogen atoms. The refinement of this dataset gives positional parameters (rms deviation $=0.008 \AA$ ) which are in very good agreement with the Elettra dataset measured at $120 \mathrm{~K}$ and refined using the Independent Atom Model (IAM) method. The equivalent atomic displacement parameters of the non-hydrogen atoms are in general 7 to $10 \%$ lower than those of the Elettra data set ( $\mathrm{rms}$ deviation $=0.002 \AA$ ). This discrepancy is larger than was found in the comparative study by Fugel et al. (2018; 2-5 \%), which is most probably due to the lower resolution of the Elettra data set compared to the data sets used in the Fugel study.

Structural drawings have been made using OLEX2 (Dolomanov et al.; Fig. 3) and MERCURY (Macrae et al., 2020: Figs. 8, 13, 14). All data plots have been made using the Python MATPLOTLIB library (Hunter, 2007).

\section{Results}

\subsection{Thermal expansion evolution with temperature}

Fig. 1 shows the evolution of the cell parameters as a function of temperature and the data collection number.
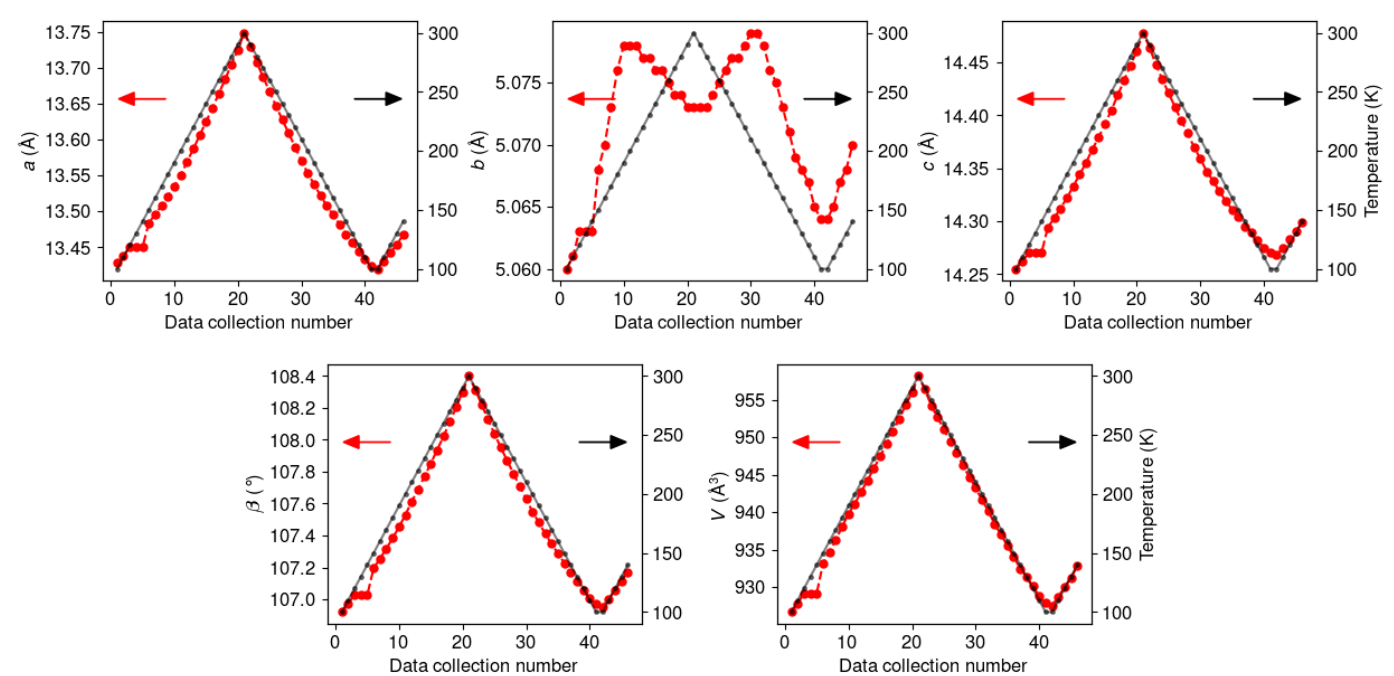
Figure 1 Evolution of cell parameters (red markers, left axis) of the structure of BTBT-C4OH as a function of data collection number. The temperature of each data collection is displayed by tiny black dots projected on the right axes.

Close to linear temperature dependent evolutions are observed for the $a$ and $c$ axes, as well as for the $\beta$ angle and the volume. The $b$-axis however shows at approximately $200 \mathrm{~K}$ a crossover from positive to negative thermal expansion. This behaviour is reproducible when cooling again through the transition temperature. A clear hysteresis can not be determined, since the crossover appears to span approximately $15-20 \mathrm{~K}$, i.e. it is not a very sharp transition with clear jumps in one or more cell parameters. A similar behaviour was found for the positive to negative thermal expansion transitions reported by Negi et al. (2018) where the crossover appeared to span almost $50 \mathrm{~K}$. These very smooth changes would indicate rather a second-order phase transition, but isosymmetric phase transitions are necesserily first-order (Christy, 1995).

In a monoclinic space group setting with the $b$ axis as unique axis it is only the expansion along the $b$ axis which represents the true expansion, the expansions along the $a$ and $c$ axes being correlated to changes in the $\alpha$ and $\gamma$ angles. The two expansions perpendicular to the $b$ axis are found by solving the eigenvalue problem of the strain matrix. Fig. 2 gives the expansions along the three perpendicular directions $\mathrm{X} 1, \mathrm{X} 2$, and $\mathrm{X} 3$ as a function of temperature.

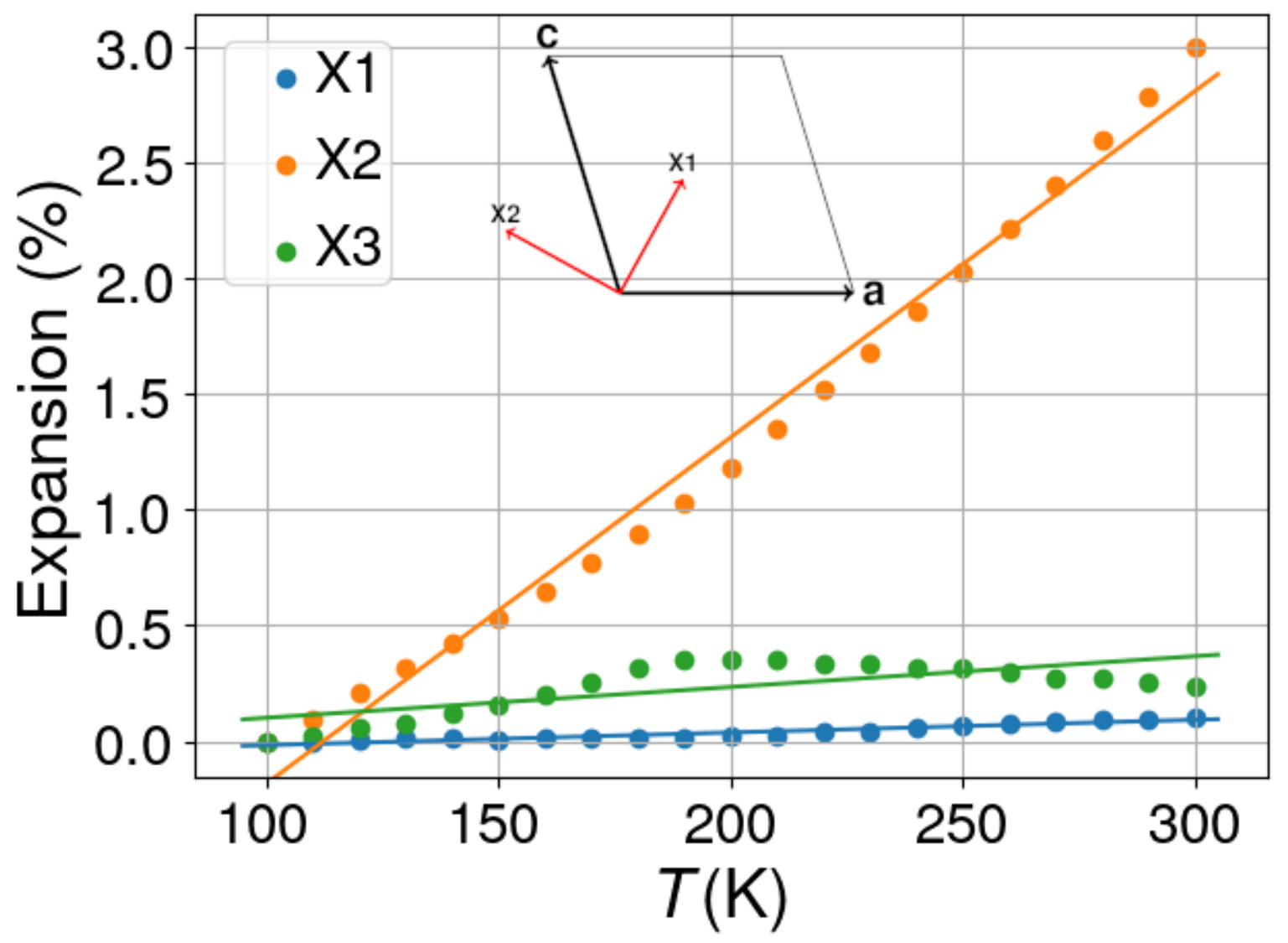


Figure 2 Percentual thermal expansion in the structure of BTBT-C4OH relative to $100 \mathrm{~K}$ along the three perpendicular directions in the monoclinic system corresponding to the eigenvalues of the strain matrices. The straight lines represent the result of a regression in the hypothesis of linear thermal expansion. The inset shows the orientations of the $\mathrm{X} 1$ and $\mathrm{X} 3$ axes in the orthogonal coordinate system with respect to the crystallographic axes system. Note that these orientations change slightly with temperature. The $a$ and $b$ axes are proportional to their actual lengths, whereas the $\mathrm{X} 1$ and $\mathrm{X} 2$ axes have been drawn with equal lengths for clarity.

The $\mathrm{X} 3$ axis corresponds to the $b$-axis in the crystallographic system, whereas $\mathrm{X} 1$ and $\mathrm{X} 2$ are perpendicular to each other in the $a c$ plane. The linear fits give uniaxial thermal expansion coefficients of $5.48 \mathrm{MK}^{-1}\left(R^{2}=0.8964\right), 149.50 \mathrm{MK}^{-1}\left(R^{2}=0.9876\right), 13.29 \mathrm{MK}^{-1}\left(R^{2}=0.5046\right)$ for $\mathrm{X} 1, \mathrm{X} 2, \mathrm{X} 3$, respectively, and $169.85 \mathrm{MK}^{-1}\left(R^{2}=0.9984\right)$ for the volumetric expansion coeficient between 100 and $300 \mathrm{~K}$. For this calculation - and also for the subsequent analyses - only the first 21 data collection points have been take into account. The linear approximation for the behaviour of the thermal direction along the $b$ direction is of course not justified and two new linear coefficients can be calculated between 100 and $190 \mathrm{~K}$ and 210 and $300 \mathrm{~K}$, respectively. This gives $40.36 \mathrm{MK}^{-1}$ for the temperature range $100-190 \mathrm{~K}\left(R^{2}=0.9807\right)$ and $-12.41 \mathrm{MK}^{-1}\left(R^{2}=0.9754\right)$ for the temperature range $200-300 \mathrm{~K}$. The negative thermal expansion for the latter temperature range is approximately 20 times smaller than the one reported in the BTBT-C6OH (Van der Lee et al., 2018), whereas the positive thermal expansion along X2 is more than 3 times smaller than the largest positive thermal expansion in BTBT-C6OH. The change of the sign of the thermal expansion does not involve a space group change.

\subsection{Geometrical evolution of the BTBT-C4OH structure with temperature}

The molecular structure of BTBT-C4OH (Fig. 3) presents the same characteristics as other symmetric or asymmetric funtionalized BTBT systems, i.e. an essentially planar BTBT core with rms deviations between 0.001 and $0.002 \AA$ for all temperatures satisfying the $\chi^{2}<5$ test for planarity, and, in the case of chain-like functions, subtending angles between chains and the planar core system lying between 31 and $32^{\circ}$.

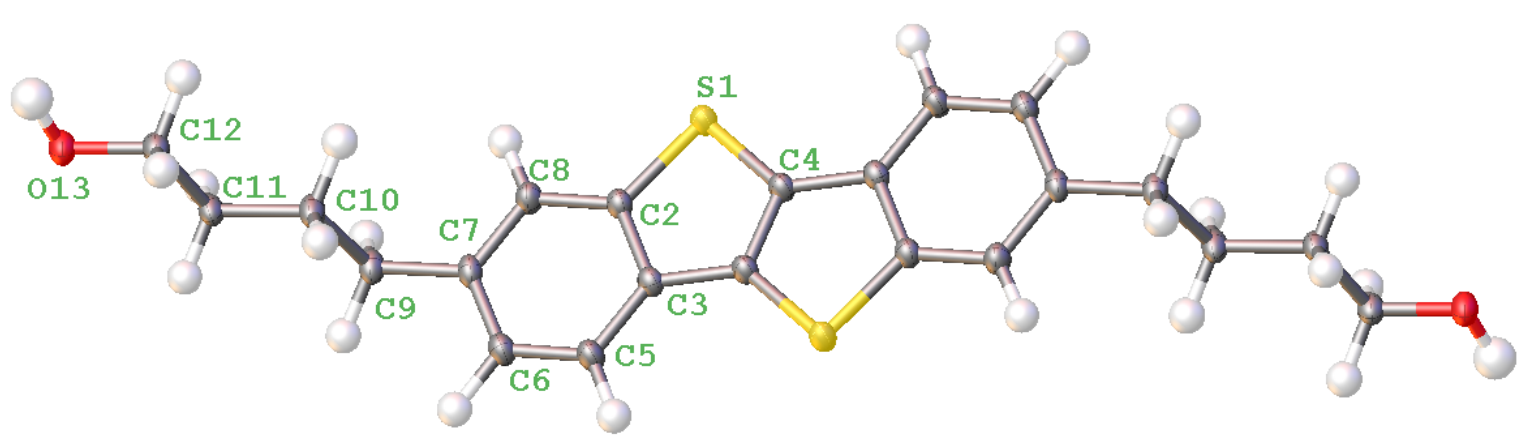


Figure 3 ORTEP-style plot of the structure of BTBT-C4OH. The independent atoms in the molecule are labelled. The atoms on the each side of the molecule are related by an inversion centre to those on the other side. Atomic displacement ellipsoids and spheres (for the $\mathrm{H}$ atoms) are at the $30 \%$ probability level.

The geometrical characteristics of the infinite hydrogen bond chains along the $b$-axis follow closely the expansion along that axis. Fig. 4 gives the intermolecular $\mathrm{O} \cdots \mathrm{O}$ distance and $\mathrm{O} \cdots \mathrm{O} \cdots \mathrm{O}$ angle as a function of temperature. The vector of the $\mathrm{O} \cdots \mathrm{O}$ distance is not completely aligned with the $b$-axis and therefore the distance only levels off after the transition temperature, whereas the $\mathrm{O} \cdots \mathrm{O} \cdots \mathrm{O}$ angle after an initial increase decreases with temperature.

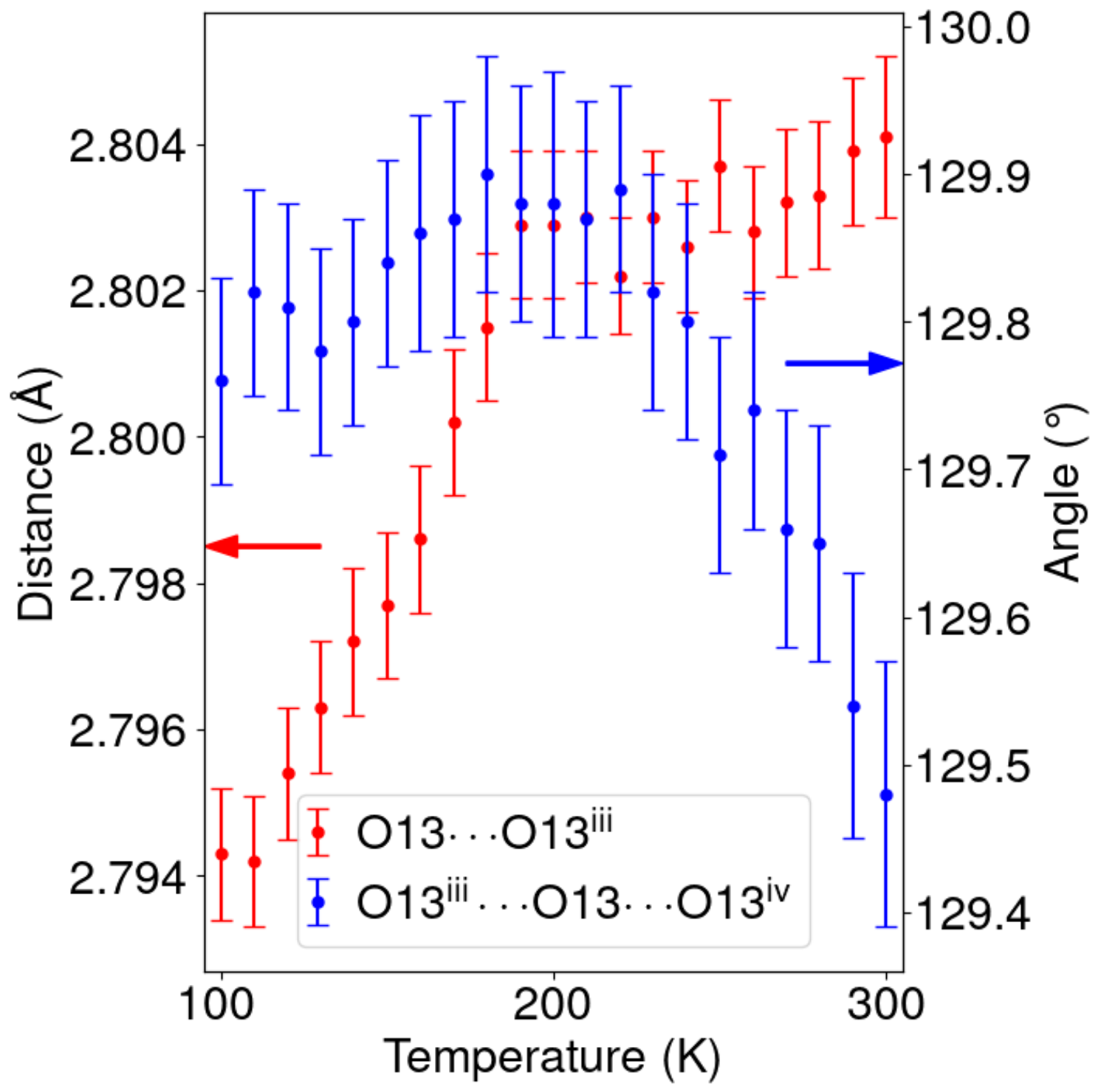

Figure 4 Distance and angle changes in the hydrogen bonded infinite chain in BTBT-C4OH along the $b$-axis. Symmetry codes: (iii) $2-x, y-1 / 2,-z+5 / 2$; (iv) $2-x, y+1 / 2,-z+5 / 2$. 
The C-C distances within the BTBT core are nearly independent of the temperature, whereas the two different S-C distances contract with temperature, the S1-C2 distance only after the transition temperature, but the S1-C4 distance over the complete temperature range (Fig. 5). Changes in aromaticity in meta-carboxyphenylammonium phosphite across a first-order isosymmetric phase transition have been observed before by Bendeif et al. (2009). The shortening of the two bonds may be related to the fairly important atomic displacement of $\mathrm{S} 1$ in a direction perpendicular to the BTBT core plane (see section 4.3).

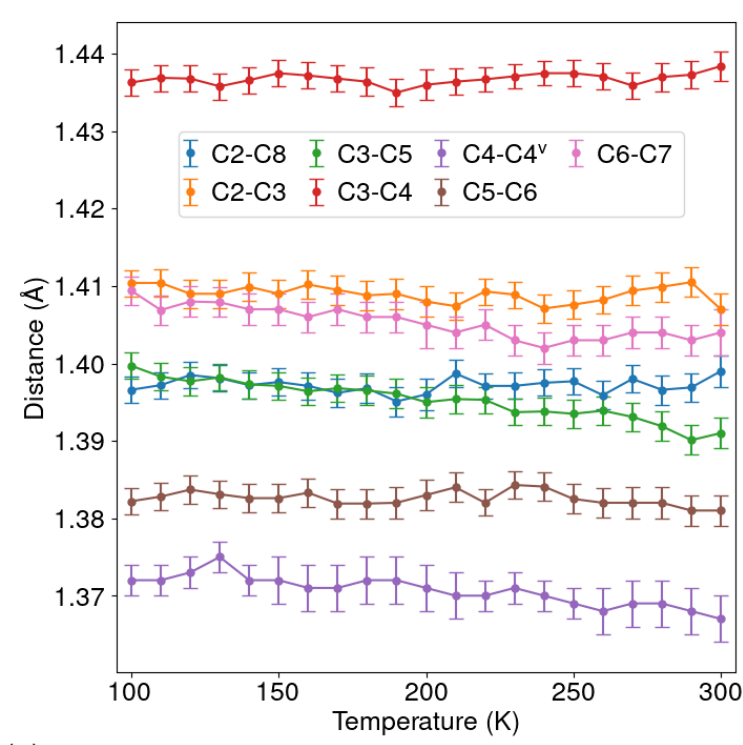

(a)

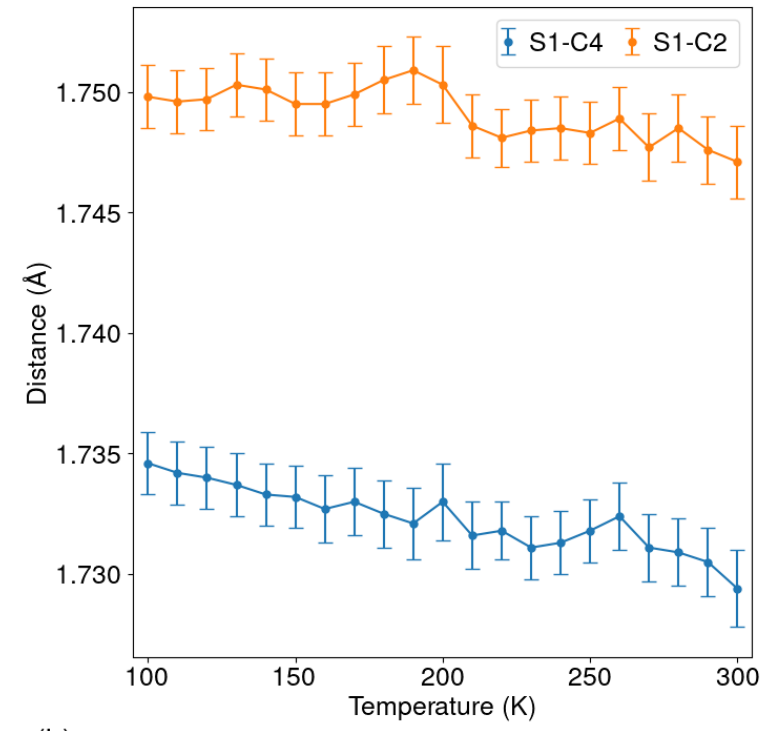

(b)

Figure 5 (a) C-C and (b) S-C distances as a function of temperature in the structure of BTBT$\mathbf{C 4 O H}$; symmetry code: (v) 1-x,1-y,1-z. Since the third eigenvalue of the librational tensor $\boldsymbol{L}$ is equal to zero for all temperatures (Fig. 11), no corrections to the distances due to librational motions have been calculated.

The individual $x, y$, and $z$ coordinates of the non-hydrogen atoms do not show very abrupt changes with temperatures, but anomalies around the cross-over from positive to negative thermal expansion can be detected for notably the $z$ coordinates of S1, C12 and O13 (Fig. S1-S13).

\subsection{Evolution of the atomic displacement parameters of BTBT-C4OH with temperature}

The temperature evolution within the structure can also be followed by plotting the $U_{\text {eq }}$ values of the atoms of the molecule as a function of temperature (Fig. 6). A clear signature of the phase transition at $200 \mathrm{~K}$ is found by a change of slope and a sudden drop of the $U_{\text {eq }}$ value for all atoms. The largest $U_{\text {eq }}$ values are - not surprisingly - found for the terminal $\mathrm{OH}$ group. If the atomic displacement would be harmonic in the classical regime, a linear relationship with temperature would be expected which extrapolates to zero at $T=0 \mathrm{~K}$. The linear relationship is indeed observed in both the LT $(<200 \mathrm{~K})$ and the HT (> $200 \mathrm{~K}$ ) phase, but the $U_{\mathrm{eq}}$ values do not extrapolate to zero in neither the low nor the high temperature regimes. The positive extrapolated values at $0 \mathrm{~K}$ in the LT phase is usually 
indicative of unresolved static disorder or strongly negative anharmonicity, whereas the negative intercepts in the HT phase would indicate unresolved positive anharmonicity (Bürgi et al., 2000). In this context positive anharmonicity refers to decreasing vibration frequencies and positive thermal expansion, whereas negative anharmonicity refers to increasing vibration frequencies and negative thermal expansion (Evans et al., 1997). This would be in contradiction with the observed crossover from positive to negative thermal expansion in BTBT-C4OH, but it should be remembered that this crossover is only observed for the $b$-axis. A refinement with $3^{\text {rd }}$ order non-harmonic atomic displacement parameters for the non-hydrogen atoms was performed using JANA2006 (Petricek et al., 2014) for the 100 and $300 \mathrm{~K}$ data sets. The refinement converged smoothly with slightly better $R$ factors than with $2^{\text {nd }}$ order atomic displacement parameters only, but most of the $3^{\text {rd }}$ order terms were within 3 times their standard uncertainties. Only a few parameters were significant up to 7 times their standard uncertainty.

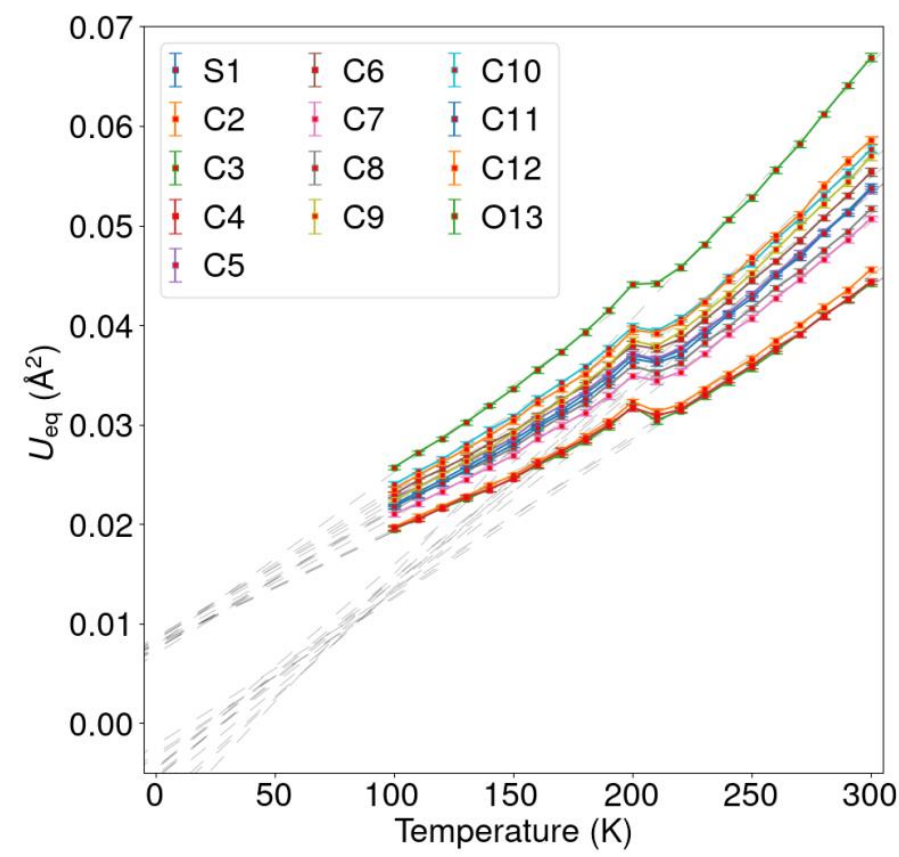

Figure $6 U_{\mathrm{eq}}$ values of non-hydrogen atoms in the structure of BTBT-C4OH as a function of temperature. For the atom labeling see Fig. 3. The dashed lines are the results of linear fits to the data points in the low and the high temperature regime, respectively.

A more detailed investigation of the temperature dependence of the vibrational characteristics of the BTBT-C4OH molecule can be obtained by analyzing the atomic displacement parameters (ADP's) of each non-hydrogen atom of the BTBT-C4OH molecule. The ADP is a 3x3 tensor, represented in 3D space as an ellipsoid, for which the 3 eigenvalues give the size of the three ellipsoid principal axes and the eigenvectors their directions in a Cartesian coordinate system. The anisotropy of the ellipsoid is usually expressed as the ratio of the minimum ellipsoid axis (minimum eigenvalue) to the maximum 
ellipsoid axis (maximum eigenvalue). The change of anisotropy of the displacement ellipsoid through phase transitions has been occasionally determined in the literature e.g. by Brown et al. (1994) in the mineral Co-Åkermanite or by Smeets et al. (2011) for a $\mathrm{Cu}^{\mathrm{II}} \mathrm{N} 6$ and $\mathrm{Zn}^{\mathrm{II}} \mathrm{N} 6$ complex.

Here the ellipsoid anisotropy index is used which has the additional advantage that changes in the three axes are monitored instead of two. It is defined as

$\mathrm{IAC}=\mathrm{X} 1 / \mathrm{X} 2-\mathrm{X} 2 / \mathrm{X} 3$

for the quantification of rods, plates, and intermediate forms in 3D ellipsoidal geometries (Doube, 2015 ) with $X 1, X 2$, and $X 3$ the lengths of the principal axes of the ellipsoid, and $X 1<=X 2<=X 3$. The ellipsoid anisotropy index (IAC) ranges from -1 for very oblate ellipsoids to +1 for strongly prolate ellipsoids.

Fig. 7 shows the ellipsoid anisotropy indices of the atoms of the BTBT core (a) and those of the side chains (b).
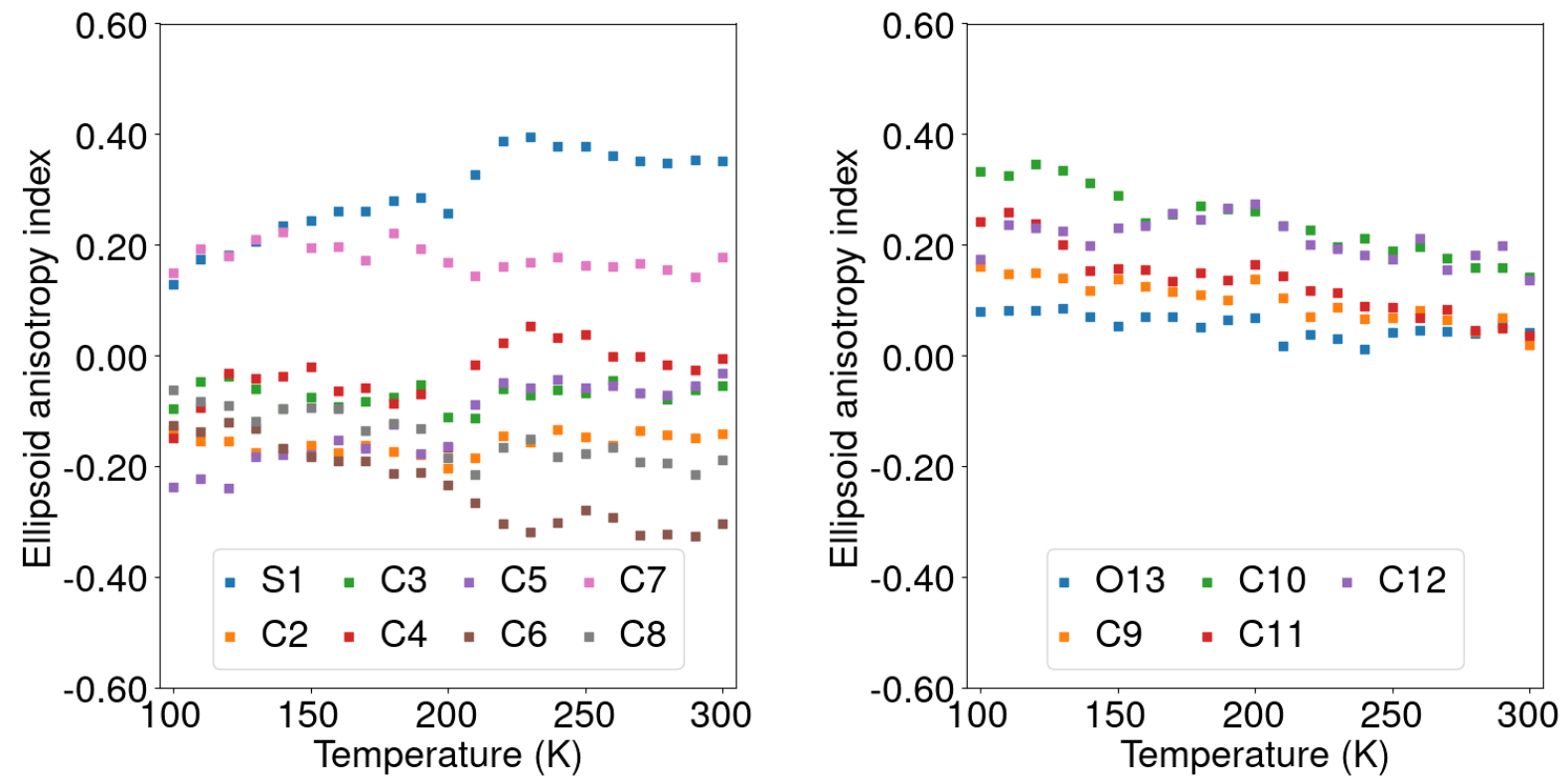

Figure 7 Ellipsoid anisotropy index as a function of temperature for the atom in the BTBT core (left) and for those in the side chains (right) 
Although the scatter is relatively important, it appears that the phase transition can also be detected in the anisotropy evolution of the atoms of the BTBT core, some atoms becoming more oblate, and others more prolate. The values of the ellipsoid anisotropy index for all atoms vary between -0.30 and 0.40 , meaning that the anisotropy is only moderate. In the HT phase the anisotropy is much more dispersed than in the LT phase. In the side chains there is a general tendency that the atoms become more oblate with temperature, but with less pronounced behavior at the phase transition. Interestingly, the largest positive - prolate - anisotropy is found for the sulfur atom, the heaviest atom of the molecule. The anisotropy of the atomic displacement ellipsoid increases significantly through the phase transition, where the eigenvector of the longest principal axis is close to perpendicular to the plane of the BTBT moiety for all temperatures. These results are confirmed by the Hirshfeld atomic refinement, which gives in principle more 'neutron-like' atomic displacement parameters.

A similar trend is observed by plotting the three eigenvalues of the atomic displacement ellipsoid as a function of temperature for each atom (Fig. S2). As was found for the $U_{\text {eq }}$ values, the eigenvalues alos extrapolate to either negative or positive values at $0 \mathrm{~K}$. Subtle changes can be detected by analyzing the directions of the principal axes. Fig. 8 shows the best overlay - made with MERCURY (Macrae et al., 2020) - of the BTBT-C4OH molecule at $200 \mathrm{~K}$ and $210 \mathrm{~K}$, i.e. close to the temperature where the negative discontinuity in the eigenvalues is found. The positional overlay seems nearly perfect and the ellipsoids at the $90 \%$ probability level have visually the same size. However notable changes in the directions of the principal axes of the displacement ellipsoids of the atoms in the BTBT core are observed, whereas the directional changes for the ellipsoids of the side chain are negligible. Fig. 9 shows the temperature evolution of the angles between the atomic displacement ellipsoid principal axes and the normal vector of the least-squares BTBT plane for the S1 atom. Fig. S3 contains the same data for the other atoms. 

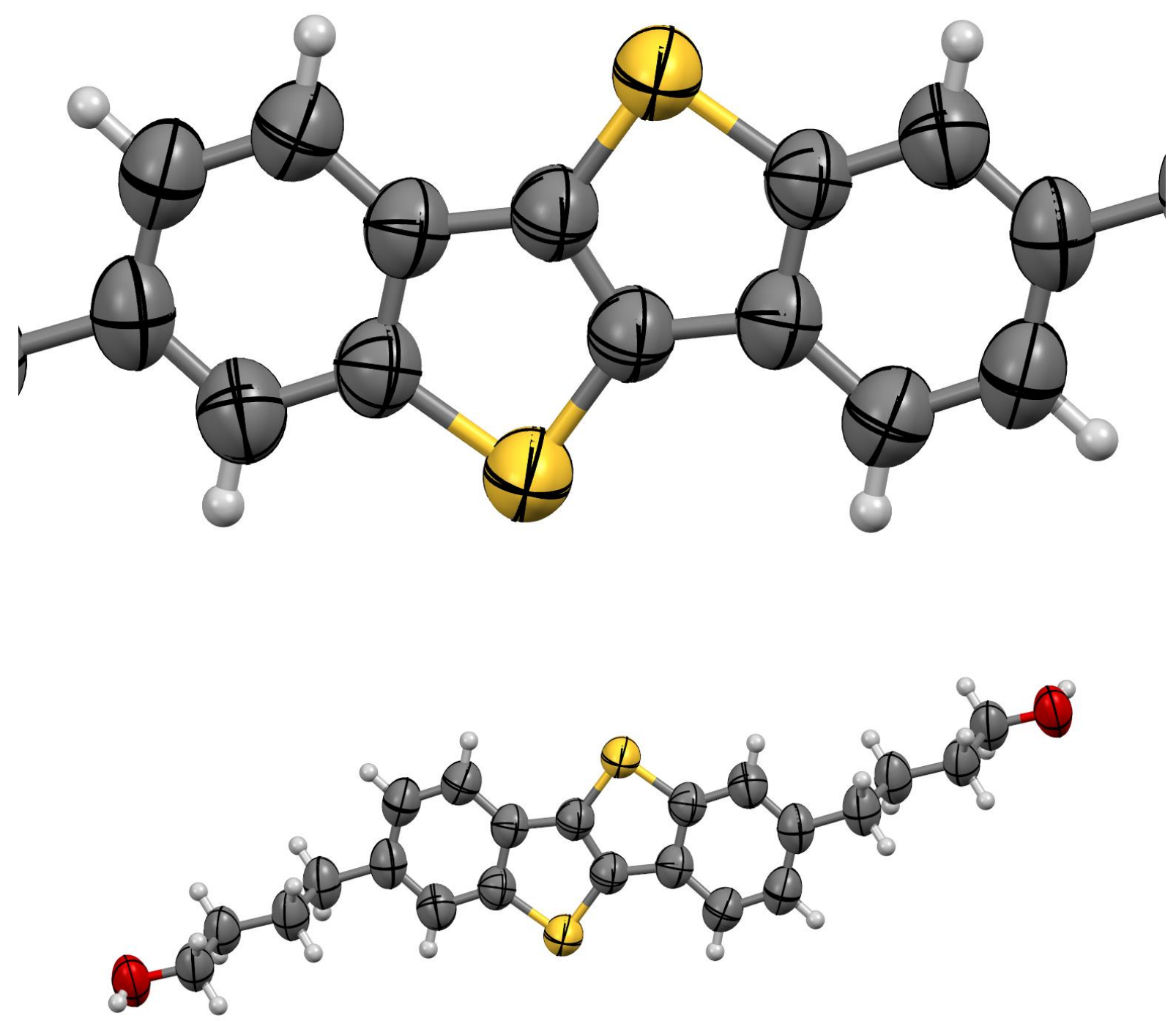

Figure 8 Molecular overlay of the BTBT-C4OH structures at $200 \mathrm{~K}$ and $210 \mathrm{~K}$ with the atomic displacement ellipsoids at the $90 \%$ probability level. Hydrogen atoms are shown as spheres with $0.2 \AA$ radius. The bottom figure shows the best overlay of the complete molecules as calculated by MERCURY, whereas in the top view only the BTBT core is displayed and zoomed out for better visibility. 

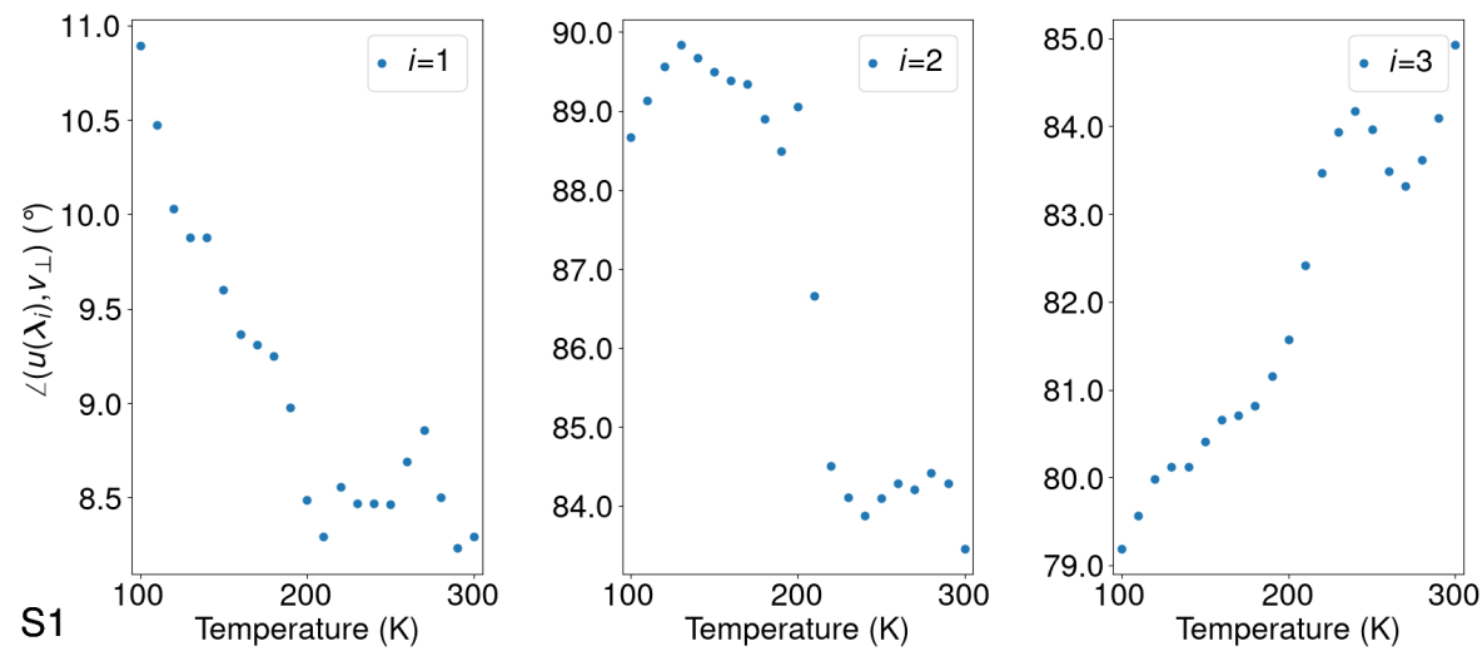

Figure 9 Temperature evolution of the angle between the normal $\boldsymbol{v}_{\perp}$ of the BTBT least-squares plane and the atomic principal displacement vectors $\boldsymbol{u}\left(\lambda_{i}\right)$ of S1 where $i=1,2,3$ with eigenvalues in decreasing order.

There is considerable scatter in the data points, but there is clear discontinuous behavior at the $200 \mathrm{~K}$ phase transition with saturating angle evolution for the angles corresponding to the two largest eigenvalues. The meaning of this temperature evolution can, however, not be deduced from these results.

The combined results show that the direction of largest principal axis of S1 is nearly parallel to the normal of the BTBT core least-squares plane, but that the directions of the largest principal axes of the other atoms constituting the BTBT core are far from perpendicular to the plane. It was tested whether this would lead to violations of the standard Hirshfeld rigid bond test, but that was not the case. It is well possible that more elaborated ('enhanced') Hirshfeld rigid bond tests could detect these problems (Lübben et al., 2016; Lübben, 2017). This has not been verified. However, by a comparison with the results from the HAR it was, however, concluded, that the atomic displacement eigenvector directions are most probably trustworthy (Table S2).

The general temperature evolution of the atomic displacement tensors can be obtained by plotting the quality parameters $R_{\text {eigval }}, R M S_{\text {eigvecs, }} S_{12}$, and the combined figure of merit FOM for successive data sets at $10 \mathrm{~K}$ intervals. The similarity index $S_{12}$ has been defined by Whitten \& Spackman (2006) and is connected to the overlap $R_{12}$ between two probability density function described by the $U_{i j, 1}$ and $U_{i j, 2}$ tensors with $S_{12}=100\left(1-R_{12}\right)$. Since this parameter is rather insensitive for rotational changes of the principal axes for tensors that are not very anisotropic, Sovago et al. (2014) have defined the index $R M S_{\text {eigvecs }}$ which gives the RMS deviation of principal axes of the orthogonalized $U_{\mathrm{ij}}$ tensors between the two sets. Together with which describes the overall changes of the eigenvalues a FOM can be defined as FOM $\left.=R M S_{\text {eigvecs }}+100 * R_{\text {eigval }}+\left(100-S_{12}\right)\right] / 300$. Fig. 10 shows the plots for the heaviest atom in the molecule, i.e. S1, calculated with the program SIMADP in the WINGX suite of programs 
(Farrugia, 1999).

S1

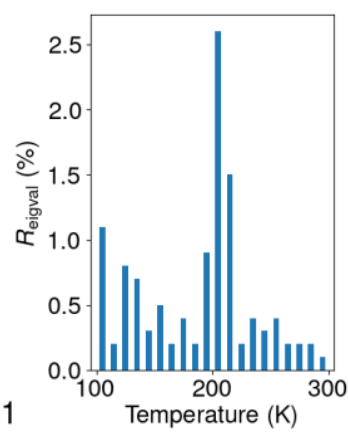

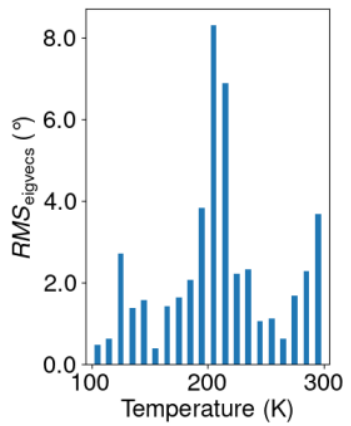
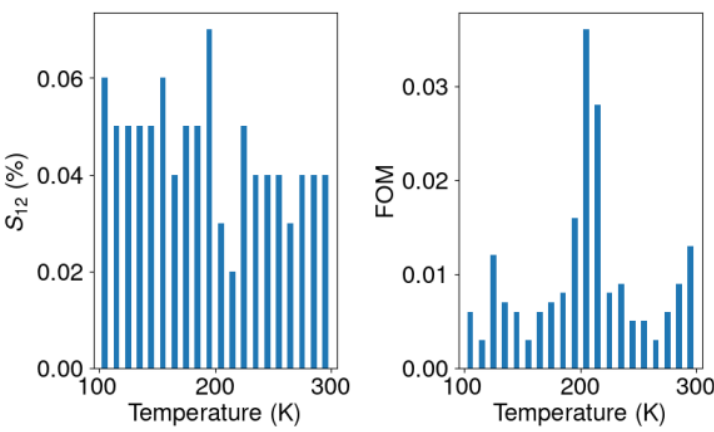

Figure $10 R_{\text {eigval }}, R M S_{\text {eigvecs, }}, S_{12}$, and the combined figure of merit FOM for the S1 ADP as a function of temperature.

The results show clearly a significant change of the ADP near 200-210 K, in line with the other results. Interestingly the rotation of the principal axes seems to be an important contribution to the change, but the overlap of the probability density functions is maximal around that temperature. The plots for the other atoms (Fig. S4) show for some of them also relatively important changes, but the discontinuities are less clear than for S1.

\subsection{TLS temperature-dependent description of the BTBT-C4OH molecule}

From the data in Fig. 6 it seems that all atoms are affected in approximately equal proportions by the phase transition. It is therefore worthwhile to analyze the correlated movements of the atoms using a semi rigid body description rather than by the individual components. According to Hirshfeld's rigid molecule test postulating that a molecule can be considered being rigid whenever the square roots of the isotropic variance of the mean-square atomic displacement (MESDU) for all non-hydrogen atoms constituting the molecule are less than $0.001 \AA^{2}$, the BTBT-C4OH molecule can be considered as being rigid at all temperatures (Hirshfeld, 1976; Hirshfeld \& Shmueli, 1972; Dunitz et al., 1988; Frey et al., 2010). The largest value, $0.00086 \AA^{2}$, is found for C6 and C12 at $300 \mathrm{~K}$. A somewhat stricter rigidity test uses Hirshfeld's rigid bond postulate as the absolute average mean displacement amplitude difference for all covalent non-hydrogen bonds within the molecule (Rosenfield et al., 1978). This value fluctuates between 0.0004 and 0.0008 for the 21 measured data sets between 100 and $300 \mathrm{~K}$, thus confirming the simpler MESDU test.

The correlated movements of the atoms within the BTBT-C4OH molecule are analyzed by the TLS (Schomaker \& Trueblood, 1968; Urzhumtsev et al., 2013) approach. In a TLS description molecules are represented as rigid bodies, whose movement is described by rotations and a translation. Thermal expansion is closely connected to the correlated movements of these rigid bodies. It can be shown 
that the amplitudes of the intramolecular modes are much smaller than those of the rigid bodies (Bürgi et al, 1988). The rigid body position itself is described by three eulerian angles and a position vector and its motion by a translation $\boldsymbol{T}$ tensor, a librational $\boldsymbol{L}$ tensor, and a coupling tensor $\boldsymbol{S}$ within the molecular inertial system $\boldsymbol{I}$. The 20 TLS parameters are derived from the observed atomic displacement parameters of the individual atoms making up the rigid body (Schomaker \& Trueblood, 1968; Dunitz et al., 1988). The program THMA11 (Trueblood, 1978) interfaced by WingX (Farrugia, 1999) was used for the calculations, taking into account the internal inversion symmetry of the molecule.
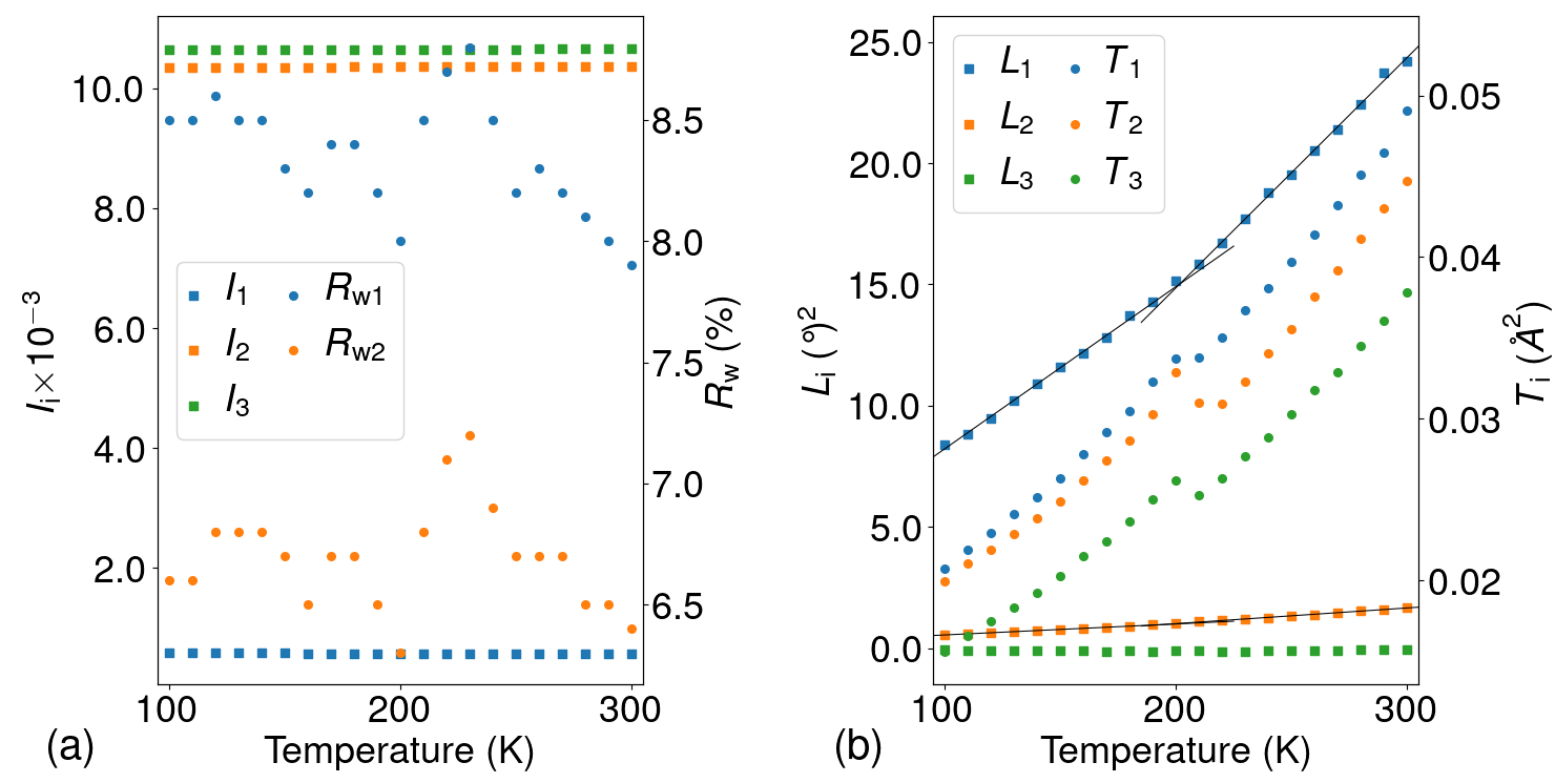

Figure 11 (a) Eigenvalues of the tensor of inertia $I$ and weighted reliability factors of the TLS model where $R_{\mathrm{w} 1}$ refers to the modelling with all $U_{\mathrm{ij}}$ 's and $R_{\mathrm{w} 2}$ with diagonal $U_{\mathrm{ii}}$ 's only. Modelling in the crystal system. $\boldsymbol{I}$ is expressed in a Cartesian coordinate system. (b) Eigenvalues of the librational tensor $\boldsymbol{L}$ and the translational tensor $\boldsymbol{T}$ (right) for a rigid-body description of BTBT-C4OH as a function of temperature. $\boldsymbol{L}$ and $\boldsymbol{T}$ are in the inertial system $\boldsymbol{I}$. The temperature dependence of the $L_{1}$ and $L_{2}$ eigenvalues was subjected to a linear regression in the intervals $100-190 \mathrm{~K}$, and $210-300 \mathrm{~K}$, respectively. The results are shown as straight solid lines.

Fig. 11 gives the eigenvalues of $\boldsymbol{I}, \boldsymbol{L}$ and $\boldsymbol{T}$ as a function of temperature; the coupling tensor $\boldsymbol{S}$ is equal to the zero tensor for all temperatures. The tensor of inertia $\boldsymbol{I}$ gives an idea how the mass is distributed in the rigid body. The eigenvector corresponding to the smallest eigenvalue of $\boldsymbol{I}, I_{1}$, has an angle mismatch of approximately $5^{\circ}$ with the crystallographic $c$-axis and is thus approximately aligned with the long molecular axis of BTBT-C4OH.

Since the three eigenvalues of $\boldsymbol{I}$ are essentially independent of the temperature, this shows that the internal conformation of the molecule hardly changes as a function of temperature. The anisotropy of the eigenvalues reflects the stretched geometry of BTBT-C4OH. However, the molecule could have 
temperature independent internal degrees of flexibility by rotation about one or more internal bonds. The TLS rigid body modeling has weighted $R$ factors well below 0.10 , showing that the molecule is fairly inflexible. It was nevertheless tested whether internal flexibility could be present by dividing the molecule in different parts connected by a rotatable bond. In view of the distribution of $U_{\text {eq }}$ values (Fig. 6) maximum flexibility is expected at the end of the chain. All four different rotation bonds in the alkyl chain were considered (Fig. 3). Table 1 compares the results of the TLS refinements using the data at 100 and $300 \mathrm{~K}$ for a rigid body assumption and a semi-rigid body hypothesis.

Table $1 R_{\mathrm{w} 1}$ and $R_{\mathrm{w} 2}$ factors for the TLS refinements of the BTBT-C4OH molecule at $100 \mathrm{~K}$ and $300 \mathrm{~K}$.

\begin{tabular}{|l|l|l|l|l|l|l|}
\hline & \multicolumn{3}{|c|}{$100 \mathrm{~K}$} & \multicolumn{3}{c|}{$300 \mathrm{~K}$} \\
\hline & $R_{\mathrm{w} 1}$ & $R_{\mathrm{w} 2}$ & $L\left({ }^{\circ 2}\right)$ & $R_{\mathrm{w} 1}$ & $R_{\mathrm{w} 2}$ & $L\left({ }^{\circ 2}\right)$ \\
\hline Rigid & 0.085 & 0.066 & - & 0.079 & 0.064 & - \\
\hline C9-C10 & 0.057 & 0.042 & $36(7)$ & 0.053 & 0.037 & $84(18)$ \\
\hline
\end{tabular}

The TLS model is composed by either a completely rigid body or a semi-rigid body with a flexible C9-C10 bond. $L$ is the value of $2\left\langle\phi \lambda^{1}\right\rangle+\left\langle\phi^{2}\right\rangle$ as defined in Schomaker \& Trueblood (1998). Note that there are in total 5 other extra parameters being refined for each added rotatable bond.

For both the $100 \mathrm{~K}$ and the $300 \mathrm{~K}$ data set only the addition of a libration around the C9-C10 bond gives sensible results with an s.u. significantly smaller than the refined rotation value. For the C7-C9 bond the squared rotation was around 0.0 with an s.u. of about 12 , whereas for the C10-C11 and C11C12 bonds the s.u. diverged.

The analysis of the torsion angles in the alkyl chain as a function of temperature (Fig. 12) does not support the assumption of the $\mathrm{C} 9-\mathrm{C} 10$ rotatable bond as an important contribution to the internal motion of the molecule, so it is possible that the drop in $R_{\mathrm{w}}$ factors after the addition of internal flexible torsion is only due to the addition of supplementary refinement parameters. 


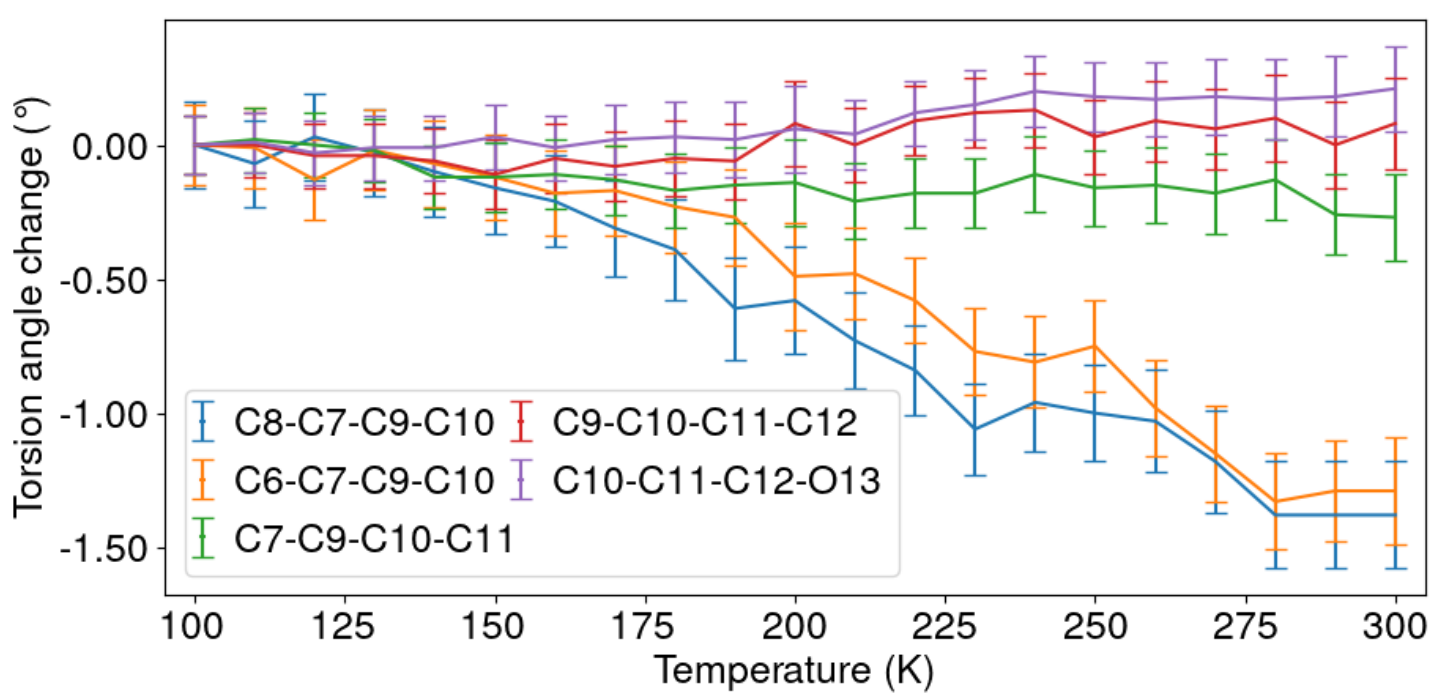

Figure 12 Torsion angles changes in the structure of BTBT-C4OH as a function of temperature. In contrast to the tensor of inertia, the librational $L_{1}$ and $L_{2}$ eigenvalues do show a dependency on temperature, and the slope of the $L_{1}$ value changes slightly at the transition temperature. The eigenvector of the largest $L_{1}$ eigenvalue, has an angle mismatch of about $5^{\circ}$ (at $295 \mathrm{~K}$ ) up to $10^{\circ}$ (at $95 \mathrm{~K}$ ) with the molecular axis that is nearly aligned with $I_{1}$. All three eigenvalues of the translational tensor $\boldsymbol{T}$ show a marked discontinuity close to $200 \mathrm{~K}$.

\section{Discussion}

The results from the temperature-dependent X-ray data collections for BTBT-C4OH show an almost unique behavior for the thermal expansion of this compound. We discuss first the structural differences and similarities between BTBT-C4OH and BTBT6-OH, before discussing the results in the wider context of anomalous thermal expansion and phase transitions.

\subsection{Comparison of the BTBT-C4OH with that of BTBT-C6OH}

BTBT-C4OH layers stack in a staggered herringbone pattern (Fig. 13), as for many other BTBT related compounds. A database survey of related compounds was presented in the supplementary material of Van der Lee et al. (2018). 


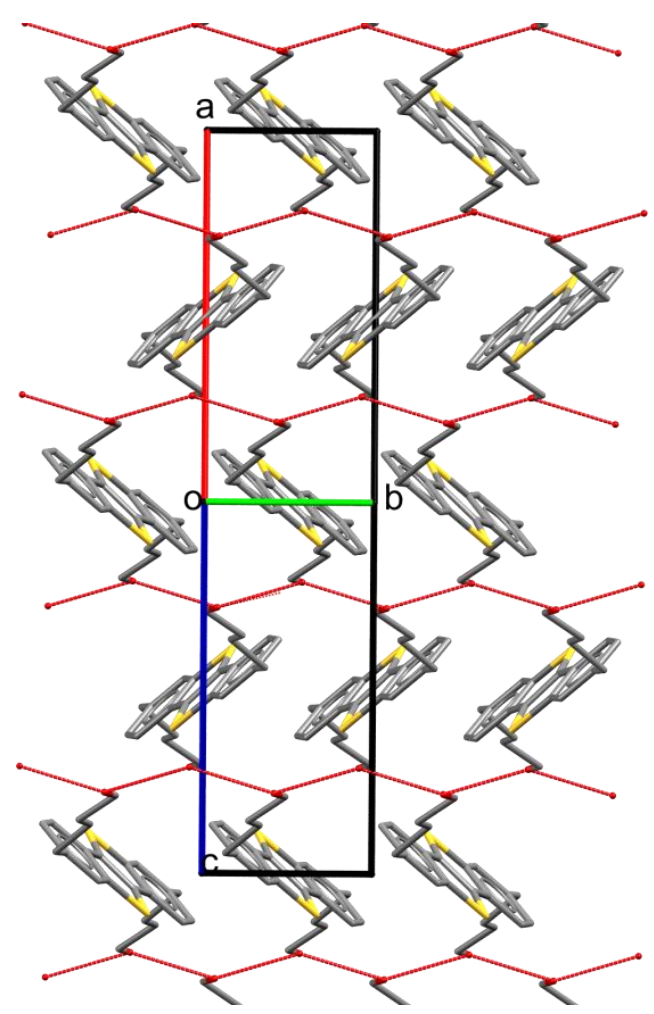

Figure 13 Projection of the BTBTC4-OH structure along <101>. Hydrogen atoms have been omitted for clarity. Hydrogen bonds are drawn as dashed red lines.

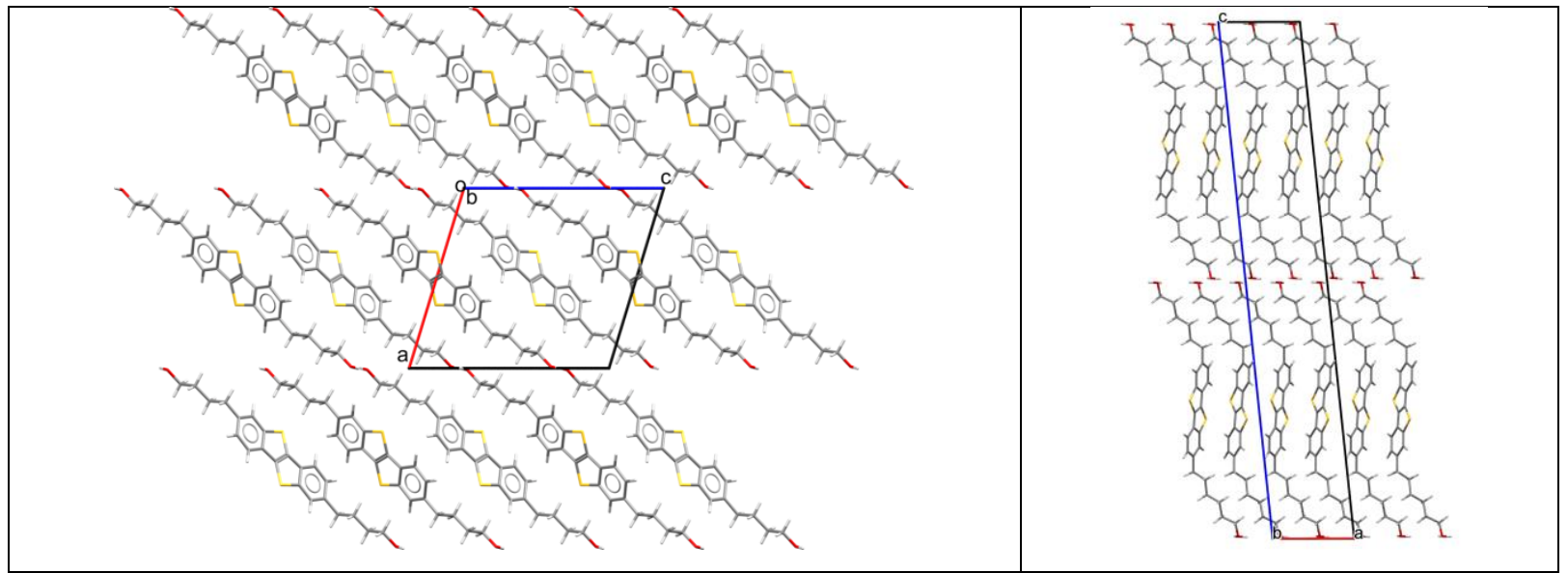

Figure 14 Projections of structures of BTBT-C4OH (100 K, left) and BTBT-C6OH (95 K, right) along $b$. 
Fig. 14 compares the stacking of the BTBT-C4OH molecules with that of BTBT-C6OH along the $b$ axis. The $b$-axis is the unique axis in both structures, since BTBT-C6OH crystallizes as BTBTC4OH in a monoclinic space group, $P 2_{1} / c$ for BTBT-C4OH and $I / a$ for BTBT-C6OH. In both structures the molecules stack in successive layers which by themselves are packed in a herringbone type arrangement. The layers are separated by a hydrogen-bonded network of infinite $\mathrm{C}^{1}{ }_{1}(2)$ chains along the $b$-axis in BTBT-C4OH, and along the $a$-axis in BTBT-C6OH. The chains are disordered in the latter case, but completely ordered in the former case.

The herringbone stacking in BTBTC4-OH does not generate, however, close S $\cdots \mathrm{S}$ contacts which are responsible for the conducting behavior of other BTBT based organic compounds, since the BTBT cores from neighboring molecules are largely displaced with respect to each other, in contrast with the aligned arrangement in the conducting structures such as BTBT-C6OH. Weak interaction between neighboring molecules occurs therefore primarily between sulfur atoms with butyl chains by relatively short $\mathrm{S} \cdots \mathrm{C}$ distances. The contact distances evolve all positively with temperatures (Fig. 15a), but no clear transition can be observed at the cross-over temperature from positive to negative thermal expansion. The same is true for the temperature evolution of the fractional coordinates of the non-hydrogen atom in the structure (Fig. S1). In contrast with the structure of BTBT-C4OH some of the intermolecular close contact distances in the structure of BTBT-C6OH are closely correlated with the thermal expansion along the crystallographic axes.

The BTBT herringbone angle, which is mostly between 50 and $60^{\circ}$ in the conducting BTBT based structures (Van der Lee et al., 2018), is in BTBT-C4OH close to $90^{\circ}$. Fig. 15b gives the herringbone angle $\sigma_{\mathrm{h}}$ in the structures of BTBT-C6OH and BTBT-C4OH. Note that the scales of $\sigma_{\mathrm{h}}$ for BTBTC6OH and BTBT-C4OH are very different, but that the evolution with temperature is very similar. The change of slope for BTBT-C4OH is at the same temperature as the cross-over from positive to negative thermal expansion. The change of slope of the herringbone angle in the structure of BTBTC6OH corresponds to a change of slope of the negative thermal expansion, being much higher in the high temperature regime than in the low temperature regime.
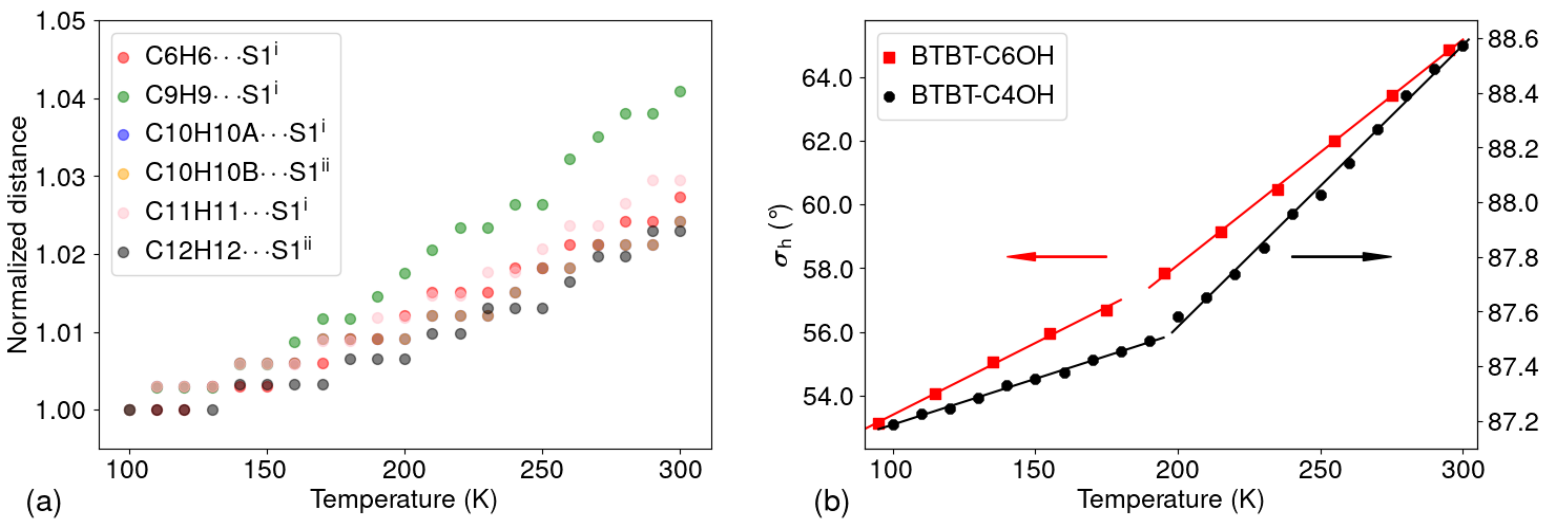
Figure 15 (a) Temperature evolution of relatively short intermolecular contacts in the structure of BTBT-C4OH. Symmetry codes: (i) $x,-y+3 / 2, z$; (ii) $x,-y+1 / 2, z$. (b) herringbone angle $\sigma_{\mathrm{h}}$ as a function of temperature in the structures of BTBT-C6OH (red filled squares) and BTBT-C4OH (black filled circles).

\subsection{Negative thermal expansion and diffuse crossover phase transition}

As was outlined by Coates \& Goodwin (2019) several mechanisms can be distinguished that may be responsible for volumetric and or axial negative thermal expansion. Phonon mediated NTE as found in open structures with directional covalent bonding favors a maximal temperature range in which the NTE occurs, although the NTE is not necessarily very strong. NTE is usually the strongest when electronic transitions are involved, but they span usually a small temperature range, e.g. through a phase transition. Some mechanisms responsible for NTE in molecular materials which are bonded by supramolecular interactions have been described by Liu et al. (2018). They distinguish for this type of materials NTE caused by conformational changes of the molecules with temperature and NTE caused by rearrangement of molecules. It is clear that conformational changes are not responsible for the

NTE in BTBT-C4OH and BTBT-C6OH and the influence of internal molecular rearrangement seems also to be marginal.

From a phenomenological point of view the thermal expansion in a molecular organic compound should be the strongest in the direction of the weakest interactions such as van der Waals type interactions and weakest in the direction of the strongest intermolecular interactions, such as strong directional hydrogen bonds and even negative in the presence of chain or layer-like covalent bonds (Lifshitz, 1952). In the case of BTBT-C4OH the largest expansion (X2, Fig. 2) is in the ac plane approximately perpendicular to the long molecular axis, i.e. in the direction where only Van der Waals type interactions are present or at the most very weak $\mathrm{S} \cdots \mathrm{C}$ interactions. The two much weaker expansions are in the $\mathrm{X} 1$ and $\mathrm{X} 3$ directions, where $\mathrm{X} 3$ is parallel to the zigzag hydrogen bond $\mathrm{C} 11$ chain, holding together stacked BTBT-C4OH molecules in the same orientation in an indirect fashion, and where X2 is approximately aligned with the long molecular axes holding together molecules in alternate orientations on both sides of the interlayer gap which is parallel to the $b c$ plane. Two factors are related to the relative incompressibility in the $\mathrm{X} 2$ direction: the stiff covalent bonds in the direction of the long molecular axis and the mean direction of the strong bifurcated $\mathrm{OH}^{\cdots} \mathrm{OH} \cdots \mathrm{OH}$ interaction which is aligned with $\mathrm{X} 2$. It is interesting to note that the very strong negative axial thermal expansion in BTBT-C6OH is not along the direction of the disordered zigzag chains, but in the mean direction of the $\mathrm{S} \cdots \mathrm{C}$ interactions between adjacent BTBT cores in alternate orientations. The primary mechanism of the negative uniaxial thermal expansion along $b$ in BTBT-C4OH is, however, not different than that in BTBT-C6OH (Fig. 6 in van der Lee et al., 2018) and pentacene 
(Fig. 4 in Haas et al., 2007). It originates from a compensated steric hindrance between the herringbone-packed molecules, and is caused by the increasing librational motion of the molecules along the long molecular axis together with the increasing herringbone angle with temperature.

It was shown by DFT based ab initio molecular dynamics modeling that the low-temperature structure of BTBT-C6OH consists of two distinct structural phases $\boldsymbol{\alpha}$ and $\boldsymbol{\beta}$ of which one is dominant below a supercritical-like transition temperature. Beyond the transition temperature the $\alpha$ and $\boldsymbol{\beta}$ phases dynamically exchange in a highly anharmonic mixed-domain phase which enhances the negative thermal expansion. The observed cell parameters are close to the mean values of the average crystal structures of the $\boldsymbol{\alpha}$ and $\boldsymbol{\beta}$ phases. In view of the analogy with the temperature evolution of the herringbone angle in the structure of BTBT-C6OH it is tempting to classify the structural transition in BTBT-C4OH as a first-order isosymmetric transition or diffuse crossover transition (Christy, 1995) without discontinuity in the free-energy enthalpy which leads to supercritical behavior. More insight in this transition could be gained by ab initio molecular dynamics studies, but this is beyond the scope of this paper. It is also questionable whether $a b$ initio molecular dynamics studies would be capable to predict within a reasonable time scale the correct temperature dependence of the cell parameters, in view of the small changes involved, especially compared with the changes observed in BTBT-C6OH (van der Lee et al., 2018).

Negative uniaxial thermal expansion is much larger in BTBT-C6OH than in BTBT-C4OH and in BTBT-C6OH it is negative over the entire temperature range $95-300 \mathrm{~K}$, whereas in BTBT-C4OH it is positive in the LT domain and it becomes negative in the HT domain. This is most probably related to the shorter side chains in BTBT-C4OH with respect to those in BTBT-C6OH which make the molecules considerably stiffer, so that the individual and correlated movements are less anharmonic. Pentacene (Haas et al., 2007) without side-chains has smaller positive and negative uniaxial thermal expansion than BTBT-C4OH. Conceptionally there is some analogy with the recently described scissor-like motion in imidazolium 4-hydroxybenzene carboxylate (Dwivedi et al, 2018), although in BTBT-C4OH the hydrogen bond network does not seem to play a role.

The analysis of the atomic displacement parameters $U_{\text {eq }}$ as a function of temperature shows that the mechanisms underlying the phase transition in in BTBT-C4OH are probably different than those in displacive-type isosymmetric phase transition with abrupt changes in the cell parameters. Whereas the discontinuity of $U_{\text {eq }}$ in BTBT-C4OH is negative for all non-hydrogen atoms, it is positive for the few other cases where a similar analysis was made. Bendeif et al. (2009) reported abrupt positive jumps through the isosymmetric phase transition in the $U_{\text {eq }}$ values for the atoms of both the positive cation and the negative anion in meta-carboxyphenylammonium phosphite and Chia \& Quah (2017) for the isosymmetric transition in isonicotinamide-4-methoxybenzoic acid. A similar observation was made for the atoms in a completely different system, viz. the multiferroic $(1-x) \mathrm{BiFeO}_{3-x} \mathrm{PbTiO}_{3}$ solid 
solution system, which has a ferroelectric to ferroelectric isosymmetric phase transition (Bhattacharjee et al., 2011). A possible explanation for the negative discontinuity in $U_{\text {eq }}$ values in BTBT-C4OH is the appearance of the thermally activated higher energy $\boldsymbol{\beta}$ phase at the diffuse type 0 phase transition temperature. If the $U_{\text {eq }}$ values of the atoms in the $\boldsymbol{\beta}$ phase are intrinsically lower than those of the $\alpha$ phase, then the measured values will drop, since the observed values in the HT phase are the average of the values of the individual phases.

Well-described cases of organic structures undergoing a type-0 diffuse crossover transition are very rare. Christy (1995) describes possible cases among which the majority is rather pressure-induced than temperature-induced. A possible temperature-induced type-0 diffuse transition is orthorhombic rubrene, the archetypical compound in the domain of organic semiconductors, which was reported to undergo an abrupt increase in the field-effect mobility near T $=175 \mathrm{~K}$ (De Boer et al., 2004; Podzorov et al., 2004). DSC measurements also showed a slight anomaly at the same temperature, but the X-ray diffraction measurements as a function of temperature did not give a clear phase transition i.e. no space group symmetry change or abrupt changes in the cell parameters - except for the "molecular displacement" parameter which expresses the parallel alignment of adjacent rubrene molecules and is thus related to the molecular overlap and therefore the mobility (Jurchescu et al., 2006). Interestingly, triclinic rubrene was shown to undergo an isosymmetric phase transition on pressure near $7 \mathrm{GPa}$ (Bergantin et al., 2014), but this transition is rather to be considered as displacive first-order than as diffuse first-order.

The best described temperature-induced diffuse crossover transition is that in ammonium hexafluorophosphate, $\mathrm{NH}_{4} \mathrm{PF}_{6}$ (Swainson et al., 2002), which undergoes an apparent continuous isosymmetric first-order transition at $138 \mathrm{~K}$ and a second discontinuous first-order transition at $194 \mathrm{~K}$, which is reminiscent to the succession of (supposed) phase transitions in pentacene. As in BTBTC4OH and BTBT-C6OH the primary structural parameters such as the cell metrics or the atomic coordinates do not show any apparent discontinuity at $138 \mathrm{~K}$, making it difficult to define a suitable order parameter for the transition. The discontinuous transition at $194 \mathrm{~K}$ hints, however, to the use of the displacement of the $\mathrm{NH}_{4}^{+}$cation along the monoclinic $b$-axis, for the order-parameter of the continuous transition as well. More precisely an "excess quantity" defined as the difference between the fitted displacement according to a 2-4-6 Landau potential corresponding to the $194 \mathrm{~K}$ transition and extrapolated to below $138 \mathrm{~K}$, and the observed displacement below $138 \mathrm{~K}$, was taken as the order parameter for the continuous first-order transition. The situation in BTBT-C4OH is slightly different, because no other - discontinuous - first-order transition was yet identified which would have made it more straightforward to choose an order parameter. However, in BTBT-C4OH, discontinuities are observed in the thermal parameters and also in all three eigenvalues of the $\boldsymbol{T}$ tensor. We take here the 
$U_{\text {eq }}$ value of atom $\mathrm{S} 1$ as excess quantity or order parameter $\eta$ being the difference between the extrapolated LT values of $U_{\text {eq }}$ and the observed $U_{\text {eq }}$ value. Since no phase transition is known in the HT region a simple linear fit, which was shown to be very acceptable for the atomic displacement parameters, is used to extrapolate the eigenvalues to the LT interval. Other $U_{\text {eq }}$ values could have been taken as well. Fig. 16 shows that the transition in BTBT-C4OH has a more obvious first-order character than that in $\mathrm{NH}_{4} \mathrm{PF}_{6}$ despite the absence of discontinuities in the cell parameters.
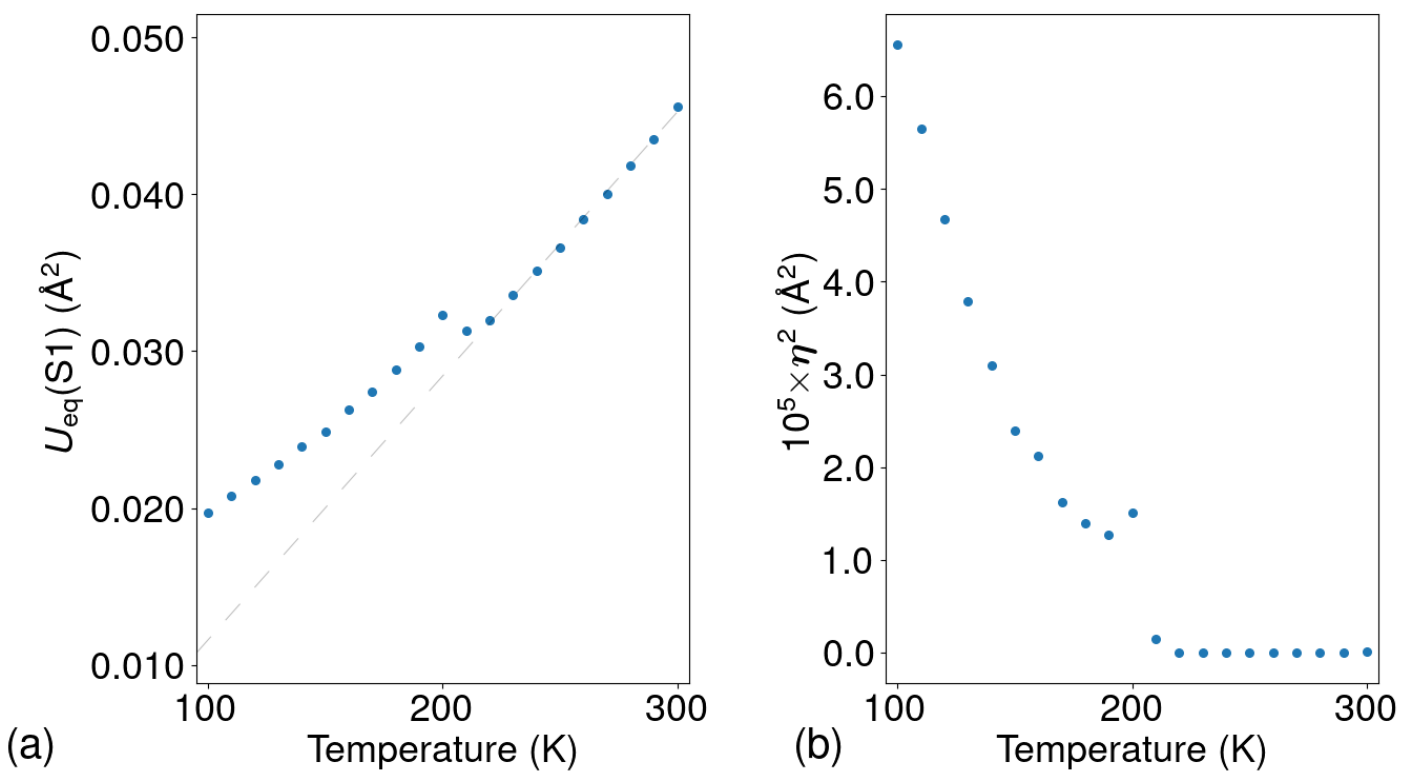

Figure $16 \quad U_{\text {eq }}(\mathrm{S} 1)$ of BTBT-C4OH as a function of temperature and linear interpolation to the low temperature regime. (b) squared excess $\eta$ value as a function of temperature.

In summary we have analyzed the structural changes of the structure of BTBT-C4OH as a function of temperature. The structural changes seem at first sight continuous, without any abrupt change of symmetry, cell parameters, intra and intermolecular contact distances and angles. The continuous change of the thermal expansion along the crystallographic $b$-axis from positive to negative is not necessarily related to a phase transition, but the abrupt changes of the atomic displacement parameters and the rigid-body eigenvalues of the $\boldsymbol{T}$ tensor at the same temperature point to a type-0 isosymmetric phase transition which is necessarily first-order. The most important changes at the phase transition on the atomic level are for the sulfur atom which shows a significant change of the direction of the eigenvectors of the atomic displacement ellipsoid but also contraction of the near-neigbour contact distances through the phase transition. From a methodological point of view it was shown that a detailed analysis of especially the atomic displacement parameters can give supplementary information about the transition which is hard to detect from the geometrical data alone. 
Acknowledgements We thank Pierre Fertey for help with the data collection carried out at the CRISTAL beamline of the Soleil synchrotron under the ЯECIPROCS BAG proposal no. 20180913.

\section{References}

Bendeif, E.-E., Lecomte, C. \& Dahaoui, S. (2009) Acta Cryst. B65, 59-67.

Bergantin, S., Moret, M., Buth, G. \& Fabbiani, F. P. A. (2014). J. Phys. Chem. C 118, 13476-13483.

Bhattacharjee, S., Taji, K., Moriyoshi, C., Kuroiwa, Y. \& Pandey, D. (2011). Phys. Rev. B84, 104116.

Brown, N.E., Ross II, C.R. \& Webb, S.L. (1994). Phys. Chem. Minerals 21, 469480.

Bürgi, H. B., Capelli, S. C. \& Birkedal, H. (2000). Acta Cryst. A56, 425-435.

Bürgi, H.-B.,Rangavittal, N. \& Hauser (2001). Ann. Helv. Chim. Acta 84, 1889-1906.

Campi, G., Lamba, D., Pifferi, A., Mande, S.C., Sarma, D.D., Sharma, S.M. \& Paolucci, G. (2015).

Eur. Phys. J. Plus, 130, 1-8

Capelli, S. C., Bürgi, H.-B., Dittrich, B., Grabowsky, S. \& Jayatilaka, D. (2014). IUCrJ 1, 361-379.

Chia, T. S. \& Quah, C. K. (2017). Acta Cryst. B73, 285-295.

Christy, A. G. (1995). Acta Cryst. B51, 753-757.

Cho, J.-m., Higashino, T. \& Mori, T. (2015). Appl. Phys. Lett. 106, 193303.

Coates, C. S. \& Goodwin, A. L. (2019). Mat. Horizon 6, 2019, 211-218.

Cliffe, M. J. \& Goodwin, A. L. (2012). J. Appl. Cryst. 45, 1321-1329.

Dautel O. et al., in preparation (2020).

De Boer, R.W. I., Gershenson, M. E., Morpurgo, A. F. \& Podzorov, V. (2004). Phys. Status Solidus A 201, 1302-1331. 
Doube, M. (2015). Front. Endocrinol. 6-15.

Dunitz, J., Schomaker, V. \& Trueblood, K. N (1988). J. Phys. Chem. 92, 856-867.

Dwivedi, B., Shrivastava, A., Negi, L. \& Das D. (2019). Cryst. Growth Des. 19, 2519-2524.

Evans, J. S. O., Mary, T. A. \& Sleight, A. W. (1997). Physica B241, 311-316.

Farrugia, L. J. (1999). J. Appl. Cryst. 32, 837-838.

Ford, S. J., Delamore, O. J., Evans, J. S. O., McIntyre, G. J., Johnson, Mark R., RadosavljevicEvans, I (2011). Chem. Eur. J. 17, 14942 - 14951.

Frey, J., Khan, S. I., Knobler, C. B., Lightner, D. A., Maverick, E. F., Phillips, D. J., Rappoport, Z. \& Trueblood, K. N. (2010). Acta Cryst. B66, 622-638.

Fugel, M., Jayatilaka, D., Hupf, E., Overgaard, J., Hathwar, V. R., Macchi, P., Turner, M. T., Howard, J. A. K., Dolomanov, O. V., Puschmann, H., Iversen, B. B., Bürgi, H.-B. \& Grabowsky S. (2018) IUCrJ 5, 32-44.

Hunter, J. D. (2007). Comp. Sci. Eng. 9, 90-95.

Kabsch, W. (2010a). Acta Cryst. D66, 125-132.

Kabsch, W. (2010b). Acta Cryst. D66, 133-144

Lausi, A., Polentarutti, M., Onesti, S., Plaisier, J. R., Busetto, E., Bais, G. Barba, L., Cassetta, A., Haas, S., Batlogg, B., Besnard, C., Schiltz, M., Kloc, C. \& Siegrist, T. (2007). Phys. Rev B76, 205203.

Liu, Z., Gao, Q., X, Chen, J., Deng, J., Lin, K. \& Xing, X. (2018). Chem. Commun. 54, 5164-5176.

Hathwar, V. R., Sist, M., Jørgensen, M. R. V., Mamakhel, A. H., Wang, X., Hoffmann, C. M., Sugimoto, K., Overgaard, J. \& Brummerstedt Iversen, B. (2015). IUCrJ. 2, 563-574.

Hirshfeld, F. L. \& Shmueli, U. (1972). Acta Cryst. A28, 648-652. 
Hirshfeld, F. L. (1976). Acta Cryst. A32, 239-244.

Iino, H., Usui, T. \& Hanna, J. (2015). Nat. Commun. 6, 6828.

Izawa, T., Miyazaki, E. \& Takimiya, K. (2008). Adv. Mater. 20, 3388-3392.

Jurchescu, O. D., Meetsma, A. \& Palstra, T. T. M. (2006). Acta Cryst. B62, 330-334

Katrusiak, A. (2001). Acta Cryst. B57, 697-704

Lifshitz, I. M. (1952). Zh. Eksp. Teor. Fiz 22, 475-486.

Lübben, J., Dittrich, B. \& Sheldrick, G. (2016). Acta Cryst. A72, s166.

Lübben, J. (2017). PhD thesis, Göttingen.

Negi, L., Shrivastava, A. \& Das, D. (2018). Chem. Commun. 54, 10675-10678.

Macrae, C. F., Sovago, I., Cottrell, S. J., Galek, P. T. A., McCabe, P., Pidcock, E., Platings, M., Shields, G. P., Stevens, J. S., Towler, M. \& Wood, P. A. (2020). J. Appl. Cryst. 53.

Petricek, V., Dusek, M. \& Palatinus, L. (2014). Z. Kristallogr. 229, 345-352.

Podzorov, V., Menard, E., Borissov, A., Kiryukhin, V., Rogers, J. A. \& Gershenson, M. E. (2004). Phys. Rev. Lett. 93, 086602.

Roche, G. H., Tsai, Y.-T., Clevers, S., Thuau, D., Castet, F., Geerts, Y. H., Moreau, J. J. E., Wantz, G. \& Dautel, O. J. (2016) J. Mater. Chem. C 2016, 6742-6749.

Rosenfield jr, R. E., Trueblood K. N. \& Dunitz, J. D. (1978). Acta Cryst. A34, 828-829.

Saha, D., Ranjan, R., Swain, D., Narayana, C. \& Guru Row, T. N. (2013). Dalton Trans. 42, 7672 7678.

Schomaker, V. \& Trueblood, K. N (1968). Acta Cryst. B24, 63-76. 
Schweicher, G., Lemaur, V., Niebel, C., Ruzie, C., Diao, Y., Goto, O., Lee, W.-Y., Kim, Y., Arlin, J.B., Karpinska, J., Kennedy, A. R., Parkin, S. R., Olivier, Y., Mannsfeld, S. C. B., Cornil, J., Geerts, Y. H. \& Bao, Z. (2015). Adv. Mater. 27, 3066-3072.

Sheldrick, G. M. (2015). Acta Cryst. C71, 3-8.

Siegrist, T., Besnard, C., Haas, S., Schiltz, M., Pattison, P., Chernyshov, D., Batlogg, B. \& Kloc C. (2007). Adv. Mater. 19, 2079-2082.

Smeets, S., Parois, P., Bürgi, H.-B. \& Lutz M. (2011). Acta Cryst., B67, 53-62.

Sovago, I., Gutmann, M. J., Grant Hill, J., Senn, H. M. Thomas, L. H., Wilson, C. C., Farrugia, and L. J. (2014). Cryst. Growth Des. 14, 1227-1239

Spek, A.L. (2009). Acta Cryst. D65, 148-155.

Swainson, I. P., Hammond, R. P., Cockcroft, J. K. \& Weir, R. D. (2002). Phys. Rev. B66, 174109, 15.

Tareyeva, E.E., Fomin, Y.D., Tsiok, E.N. \& Ryzhov, V. N. (2018). Theor. Math Phys 194, 148-156.

Trueblood, K. N. (1978). Acta Cryst. A34, 950-954.

Urzhumtsev, A., Afonine, P. V. \& Adams, P. D. (2013). Crystallogr Rev. 19, 230-270.

Van der Lee, A, Roche, G. H., Wantz, G., Moreau, J. J. E., Dautel, J. J. E. \& Filhol, J.-S. (2018).

Chem. Sci. 9, 3948-3956

Whitten, A. E. \& Spackman, M. A. (2006). Acta Cryst. B62, 875-878.

Winn, M. D. et al. (2011). Acta Cryst. D67, 235-242.

Yu, Y., Shang, R., Chen, S., Wang, B.-W., Wang, B.-W. \& Gao S. (2017). Chem. Eur. J. 23, 9857 9871. 
Yuan, Y., Giri, G., Ayzner, A. L., Zoombelt, A. P., Mannsfeld, S. C. B., Chen, J., Nordlund, D.,

Toney, M. F, Huang, J. \& Bao, Z. (2014). Nat. Commun. 5, 1-9. 